\title{
Added value of far-infrared radiometry for remote sensing of ice clouds
}

${ }^{1}$ Department of Earth and Atmospheric Sciences, Université du Québec à Montréal, Montréal, Canada

\section{Key Points:}

- Significant added value of far-infrared radiometry for ice cloud remote sensing

- Improved cloud properties retrieval in the polar regions and upper troposphere

- Extension of infrared algorithms to large optical thickness and large particles 


\section{Abstract}

10

11

Several cloud retrieval algorithms based on satellite observations in the infrared have been developed in the last decades. However these observations only cover the mid-infrared (MIR, $\lambda<15 \mu \mathrm{m}$ ) part of the spectrum, and none are available in the far-infrared (FIR, $\lambda \geq 15 \mu \mathrm{m}$ ). Using the optimal estimation method, we show that adding a few FIR channels to existing spaceborne radiometers would significantly improve their ability to retrieve ice cloud radiative properties. For clouds encountered in the polar regions and the upper troposphere, where the atmosphere is sufficiently transparent in the FIR, using FIR channels would reduce by more than $50 \%$ the uncertainties on retrieved values of optical thickness, particle effective diameter and cloud top altitude. Notably, this would extend the range of applicability of current retrieval methods to the polar regions and to clouds with large optical thickness, where MIR algorithms perform poorly. The high performance of solar reflection based algorithms would thus be extended to nighttime conditions. Since the sensitivity of ice cloud thermal emission to particle effective diameter is approximately 5 times larger in the FIR than in the MIR, using FIR observations is a promising venue for studying ice cloud microphysics and precipitation processes. This is highly relevant for cirrus clouds and convective towers. This is also essential to study precipitation in the driest regions of the atmosphere, where strong feedbacks are at play between clouds and water vapor. The deployment in the near future of a FIR spaceborne radiometer is technologically feasible, and should be strongly supported.

\section{Introduction}

Passive satellite observations have been extensively used to retrieve ice cloud radiative properties from space [e.g. Inoue, 1985; King et al., 1992; Platnick et al., 2003]. Generally, the quantities of interest are cloud optical thickness $\tau$, particle effective diameter $d_{\mathrm{eff}}$, and cloud boundaries altitudes. Contrary to active sensors such as CALIPSO [Winker et al., 2003] and CloudSat [Stephens et al., 2002] that only measure cross sections of clouds properties, passive sensors often allow imaging, which is very valuable to investigate the horizontal structure of clouds. A wide range of cloud retrieval methods have been developed, which generally take advantage of the strong variations of the ice refractive index throughout the solar spectrum and the infrared (IR) range. Methods based on solar reflection by clouds [e.g. Nakajima and King, 1990; King et al., 2004] are efficient for optically thick clouds but limited to daytime conditions. Methods based on cloud IR signature are efficient for optically thinner clouds but are limited for optical thickness greater than about 5 
[Iwabuchi et al., 2014, e.g.]. The IR methods have the advantage of being applicable indifferently for daytime and nightime, which ensures consistency of the retrieval independently of solar illumination conditions. The original IR methods were based on the split window technique [Inoue, 1985], that consists of measuring the atmosphere brightness temperature in two channels having distinct sensitivities to cloud properties. Those channels were generally chosen around 11 and $12 \mu \mathrm{m}$, ice being much less absorbing at $11 \mu \mathrm{m}$ than at $12 \mu \mathrm{m}$. Later on, the addition of supplementary channels allowed to better constrain cloud properties [e.g. Baum et al., 1994; Heli Wei et al., 2004; Yue and Liou, 2009; Wang et al., 2011, 2013]. These methods are mostly based on look-up-table approaches and retrievals are obtained by minimizing the root mean square deviation between the observations and tabulated values. More recently, a number of algorithms based on the optimal estimation method [Rodgers, 2000] were proposed [Cooper et al., 2006; Iwabuchi et al., 2014; Wang et al., 2016a]. Such methods use all available channels to find the most likely state of a cloud. They also provide a rigorous estimation of the uncertainties associated to the retrievals.

The present study is based on the optimal estimation method, and focuses on IR techniques because those can be used at night. This is critical to study clouds during the polar night, a central topic of our research. Current IR retrieval methods are not appropriate to study optically thick clouds $(\tau>10)$ and clouds made of large particles $\left(d_{\mathrm{eff}} \gtrsim 80 \mu \mathrm{m}\right)[\mathrm{Mer}$ relli, 2012; Wang et al., 2016b]. Such methods are also not suitable for the polar regions [Liu et al., 2010] due to the low thermal contrast between the surface and clouds, and because the low thermal emission of these cold areas generally results in a low signal-to-noise ratio. Those limitations are essentially due to the inherent single scattering properties of ice particles in the mid-infrared (MIR, $\lambda<15 \mu \mathrm{m}$ ), in which spaceborne radiometers such as MODIS [King et al., 1992], AIRS [Aumann et al., 2003], or AVHRR [Wang and Key, 2005] take their measurements. Yang et al. [2003] have shown that using well chosen far-infrared (FIR, $\lambda \geq 15 \mu \mathrm{m}$ ) hyperspectral channels could allow to retrieve particle effective diameters up to $100 \mu \mathrm{m}$, even in the case of optically thick clouds. This was confirmed by Merrelli and Turner [2013] who showed that the added value of FIR hyperspectral measurements is maximum for thick clouds and for large particles. FIR observations thus have the potential of observations performed in the solar spectrum, without their limitations.

Despite the acknowledged potential of FIR radiometry for retrieving ice cloud properties [e.g. Baran, 2007; Merrelli, 2012; Palchetti et al., 2016a], no satellite observations of the Earth atmosphere were performed in this spectral range since Meteor satellite program 
in the late 70's [Spankuch and Döhler, 1985]. The development of FIR spectrometers has been hindered for several reasons: 1) radiometric responsivity of existing sensors is much less in the FIR than in the MIR; 2) observations from the ground are in most locations useless since the atmosphere is essentially opaque in the FIR; 3) hyperspectral instruments in the MIR, such as AIRS or IASI [Blumstein et al., 2004], already contain much information about the atmosphere. There has been in the last decade a renewed interest for satellite missions in the FIR, though, mainly fostered by the availability of new technologies based on uncooled thermal sensors such as thermopiles [McCleese et al., 2007], pyroelectrics [Palchetti et al., 2006] and microbolometers [Ngo Phong et al., 2015]. Such sensors, when coated with gold black, have sufficient sensitivities, and are easier to deploy in space than cooled systems. The CLARREO project [Wielicki et al., 2013], for instance, aims at measuring the spectrallyresolved emission spectrum of Earth up to $100 \mu \mathrm{m}$. However, the current radiometric resolution in the FIR $(10 \mathrm{~K})$ is too low to observe clouds at short time scales. Instead, the instrument aims at providing very accurate large scale averages of the Earth radiative budget. The REFIR project [Palchetti et al., 2006], that has been recently renamed FORUM [Palchetti et al., 2016b], also intends to measure the FIR emission of the Earth, but is not specifically focused on ice cloud observation. These two projects, that are based on Fourier transform interferometry (FTIR), are ambitious and face budget issues, making their future still uncertain. Given that only a few channels $(\sim 5)$ contain the bulk of the information in most cloud retrieval algorithms [e.g. L'Ecuyer et al., 2006; Wang et al., 2016a], such high spectral resolution is not necessary for the specific application of cloud retrieval. As a consequence, an alternative resides in using narrow band radiometers rather than hyperspectral instruments. Such instruments can be more affordable, more robust and less complex than FTIR systems that require high-precision optics. Radiometers essentially trade spectral resolution for radiometric resolution. This is the route chosen for the TICFIRE project [Thin Ice Clouds in the Far-InfraRed Experiment; Blanchet et al., 2011; Libois et al., 2016a], which consists of a filter wheel IR imager with channels spanning the whole IR range from 8 to $50 \mu \mathrm{m}$. This mission currently under review at the Canadian Space Agency will be dedicated to the observation of ice clouds in the polar regions and can be viewed as a FIR extension of MODIS.

The present study investigates the potential of FIR radiometry for ice cloud radiative properties retrieval. For this, the information content of FIR radiances for a variety of cloudy scenes is estimated in the framework of the optimal estimation method. This work follows the method implemented by Wang et al. [2016a] and Iwabuchi et al. [2016] for MODIS ob- 
servations, but extends these studies to the case of FIR channels. Of particular importance is the estimation of particle effective diameter and the distinction between precipitating and non precipitating clouds. A special focus is thus put on uncertainties related to particle effective diameter retrievals and on the capacity to accurately estimate particle size for large particles. In the driest regions of the troposphere, that is the polar regions and the near-tropopause, precipitation can result in the dehydration of the atmosphere [Curry et al., 1995; Jensen et al., 1996]. This can significantly alter the radiative budget, which is essentially driven by the natural greenhouse effect of water vapor [Blanchet and Girard, 1995]. Hence cloud particle effective diameter is a critical quantity that affects the radiative budget of the Earth [e.g. Stephens et al., 2002]. Improving existing cloud retrieval algorithms could complement active technologies which provide valuable insight into clouds microphysics, formation and evolution [e.g. Austin et al., 2009; Grenier et al., 2009; Kayetha and Collins, 2016]. After providing a physical insight on the potential of FIR radiometry for studying ice clouds, Section 2 provides details on the optimal estimation framework and on the synthetic satellite instrument used in this paper. In Section 3, the information content of FIR observations is computed for two representative cases, namely a polar ice cloud and a typical tropical ice cloud. The added value of FIR is discussed in Section 4, where leads for future work and recommendations for designing a spaceborne FIR radiometer are also given.

\section{Motivation and methods}

In this section, the theoretical framework used to quantify the information content of FIR radiances for cloud properties retrieval is detailed. It is based on the optimal estimation method [Rodgers, 2000] and follows the approach of Wang et al. [2016a] and Iwabuchi et al. [2016]. Two types of ice clouds are studied in details: a polar ice cloud and a tropical ice cloud. Since no spaceborne FIR radiometer exists so far, a synthetic instrument is used, whose characteristics are mainly based on those of the Far-InfraRed Radiometer [FIRR, $\mathrm{Li}$ bois et al., 2016a], which is a breadboard for the TICFIRE mission.

\subsection{Atmospheric profiles}

The two types of clouds considered in this study are supposed to encompass most climatically relevant ice clouds encountered on Earth: a high altitude ice cloud in a tropical atmosphere, which can be either a cirrus cloud or the anvil of a convective cloud, and a ubiquitous tropospheric ice cloud during Arctic winter. Both clouds are single layer and assumed 
homogeneous. The tropical cloud is located just beneath the tropical tropopause, between 14 and $15 \mathrm{~km}$, while the polar cloud extends from 5 to $6 \mathrm{~km}$, altitudes consistent with the CALIPSO observations reported by Haladay and Stephens [2009] in the tropics and Grenier et al. [2009] in the Arctic. The atmosphere within the clouds is saturated. In the reference cases, those clouds have an optical thickness of 1.0 and a particle effective diameter of $60 \mu \mathrm{m}$, corresponding to a modified-gamma distribution with effective variance 0.1 [Petty and Huang, 2011] of severely roughened column aggregates [Yang et al., 2013]. The same distribution is assumed in the Collection 6 MODIS cloud product [Holz et al., 2016]. The characteristics of these reference cases are summarized in Table 1. The vertical profiles of temperature and water vapor mixing ratio for both atmospheres were computed from an average of 18 radiosondes taken from the IGRA database (ftp://ftp.ncdc .noaa.gov/ pub/data/igra/). For the tropical profile, 6 radiosondes measured between 1 January 2015 and 1 November 2016 were randomly taken from each of the following stations: Abidjan, Corozal Oeste, and Piarco Int. Airport. The Arctic winter profile was computed from radiosoundings measured in January or February of 2015 or 2016 at Alert, Eureka, and Resolute Bay. This profile is meant to be representative of typical winter conditions in the Canadian Archipelago. Both profiles are shown in Fig. 1.

\subsection{Instrument characteristics}

Contrary to the works of Wang et al. [2016a] and Iwabuchi et al. [2016] that were dedicated to MODIS, here we do not investigate the characteristics of a particular instrument, but rather explore the potential of a synthetic spaceborne FIR radiometer. The instrument is characterized by its number of channels, and by its noise equivalent radiance (NER). For sake of simplicity, the bands are contiguous and cover the range $6-100 \mu \mathrm{m}$, because at shorter wavelengths the contribution of solar radiation cannot be neglected in daytime conditions. The spectral responses are rectangular and the bandwidths such that all bands receive the same amount of radiance at the top of atmosphere (TOA) for the Arctic winter reference case. This equienergetic partition of the channels implies that FIR bands are much wider than MIR bands (Fig. 2). The reference configuration of the instrument is 10 bands and the NER is assumed the same for each channel, equal to $0.01 \mathrm{~W} \mathrm{~m}^{-2} \mathrm{sr}^{-1}$. This is consistent with a thermal sensor coated with gold black which has spectrally flat absorbance through the whole IR range [e.g. Ngo Phong et al., 2015], and with the absence of significant spectral differences in the transmittance along the optical path. Such characteristics are expected 
for a grating-based or filter wheel-based radiometer. The radiometric resolution is defined in terms of NER rather than in terms of noise-equivalent delta temperature (NEDT) to work at the sensor level. NEDT is helpful to compare to actual temperature measurements but it can be misleading as it depends on the temperature of the considered scene. Also, with this choice, increasing the number of bands clearly results in a decrease of the signal-to-noise ratio, which is easily understood because the sensor NER remains constant, independently of the channels configuration. In the case of gratings for instance, an increase of the spectral resolution is generally obtained at the expanse of radiometric resolution. For sake of comparison with other studies, though, Fig. 2 shows the NEDT corresponding to an NER of $0.01 \mathrm{~W} \mathrm{~m}^{-2} \mathrm{sr}^{-1}$ for different instrument configurations. The 20-band configuration is such that MIR NEDT is less than $0.2 \mathrm{~K}$, which is similar to MODIS NEDT at $250 \mathrm{~K}$. This suggests that the microbolometer-based radiometer could perform as well as MODIS sensor, except that its spectral coverage would be extended throughout the FIR.

In addition to this synthetic instrument, the method is applied to MODIS and to the TICFIRE instrument in Sect. 4.1. To this end, the ten MODIS IR channels analyzed by Iwabuchi et al. [2016] are used, and the NER for each channel is computed from Xiong et al. [2009]. The nine TICFIRE channels are defined as those of the FIRR prototype [figure 2a of Libois et al., 2016a] and cover the range 8 - $50 \mu \mathrm{m}$, and the NER is assumed to be $0.01 \mathrm{~W} \mathrm{~m}^{-2} \mathrm{sr}^{-1}$ in each channel. The spectral characteristics of all these channels are detailed in Table 2. The FIRR is very similar to the Mars Climate Sounder [McCleese et al., 2007] in terms of spectral coverage, and preliminary airborne and in situ measurements have highlighted the performances of this device, making it a realistic spaceborne instrument within the next years [ $\mathrm{Li}$ bois et al., 2016b]. The noise levels used for MODIS and TICFIRE remain constant throughout the paper.

\subsection{Physical motivation}

Most IR cloud retrieval algorithms take advantage of spectral variations of ice and water refractive index. Figure 3a shows the imaginary parts of ice and water refractive indices. It highlights the much stronger absorption at $12 \mu \mathrm{m}$ compared to $8 \mu \mathrm{m}$, which explains why in many algorithms a channel around $8 \mu \mathrm{m}$ is used along with a channel around $12 \mu \mathrm{m}$. In the FIR, ice shows a unique behavior compared to liquid water. Ice absorption indeed exhibits a significant minimum around $23 \mu \mathrm{m}$. At this minimum, the imaginary index $m_{i}$ is $2.7 \times 10^{-2}$, which is less than the value at $8.7 \mu \mathrm{m}\left(3.7 \times 10^{-2}\right)$. Since the ice absorption co- 
efficient is defined as $k_{i}=4 \pi m_{i} / \lambda$, the latter is nearly three times lower at $23 \mu \mathrm{m}$ than at $8.7 \mu \mathrm{m}$. It is also $30 \%$ less than at $3.7 \mu \mathrm{m}$, a critical channel to study optically thick clouds that is however affected by solar radiation in daytime conditions [Nakajima and King, 1990; Platnick et al., 2003]. The ice refractive index spectral features determine the spectral dependence of the extinction coefficient and single scattering albedo of ice crystals (Fig. $3 \mathrm{~b}$ and c). The reduced size parameter $\left(x=\pi d_{\mathrm{eff}} / \lambda\right)$ for wavelengths beyond $30 \mu \mathrm{m}$ is responsible for a large sensitivity of the extinction efficiency and single scattering albedo to effective diameter compared to the MIR. This makes FIR more appropriate than MIR for studying large cloud particles. In addition, the single scattering albedo is relatively high around $23 \mu \mathrm{m}$ and remains well above 0.5 for large particles. As a consequence, a significant amount of multiple scattering occurs in this range so that optically thick ice clouds are partly reflective and have an emissivity below 1 . This drastically increases the sensitivity of emitted radiance to the particle effective diameter [Yang et al., 2003], contrary to the perfect blackbody behavior observed in the MIR that hinders any information about cloud particle size. Such spectroscopic characteristic is favorable for studying cloud microphysics at the top of optically thick convective towers for instance, where standard IR algorithms show poor performance. To some extent, the FIR contains similar information to MODIS channel centered at $3.8 \mu \mathrm{m}$ used by Wang et al. [2016a], with the advantage of being unaffected by solar radiation. Note also that ice cloud optical thickness can be significantly different at 10.6, 12, 23, or $35 \mu \mathrm{m}$ (Figure $3 b$ ). For instance, a cloud with particle effective diameter of $30 \mu \mathrm{m}$ becomes opaque (i.e. optical thickness equals 5) at visible optical depth of 6.25 at $10.6 \mu \mathrm{m}, 4$ at $23 \mu \mathrm{m}$, and 7.63 at $35 \mu \mathrm{m}$. Mention of large optical thickness in the following should be considered relative to the wavelength of interest.

As shown above, the single scattering properties of ice clouds are more sensitive to particle effective diameter in the FIR than in the MIR. The spectral signature of ice clouds at TOA does not depend only on their single scattering properties, though. It also depends on the transparency of the atmosphere and on the total amount of radiation reaching the instrument. From the ground, the atmosphere is often opaque in the FIR range due to strong absorption by water vapor, so that FIR observations are generally disadvantaged compared to MIR observations. Largely for that reason, most studies dedicated to FIR radiation were taken in dry, cold or elevated locations [e.g. Turner and Mlawer, 2010; Shi et al., 2016]. On the contrary, from a satellite perspective, clouds can be observed in the FIR if the atmosphere above them is sufficiently transparent. Figure 4 shows the transmittance of the atmosphere 
above the reference clouds as a function of cloud top altitude. It shows that an ice cloud can be well observed from space up to $50 \mu \mathrm{m}$ as soon as its top is above $5 \mathrm{~km}(10 \mathrm{~km})$ for Arctic winter (tropical) conditions. These conditions are highly relevant because thin ice clouds encountered in the polar regions and tropical cirrus and convective towers often satisfy those conditions. It also shows that FIR offers an effective profiling capability throughout most of the atmosphere, by masking lower atmospheric layers and shielding the surface with increasing wavelengths in the range $17-40 \mu \mathrm{m}$.

\subsection{Optimal estimation framework}

\subsubsection{Theoretical framework}

Generally speaking, the information content of a radiance measurement depends on its sensitivity to changes in cloud properties, on the uncertainties regarding the atmospheric state, the cloud particle size distribution and shape, and on the instrumental error. The optimal estimation method [Rodgers, 2000] accounts properly for all these contributions and has been used to asses the information content of various IR instruments such as MODIS [L'Ecuyer et al., 2006] and IASI [Rabier et al., 2002]. Since the present paper focuses on cloud radiative properties, the state vector to be retrieved only includes cloud properties. Following Wang et al. [2016a], only single layer homogeneous clouds are considered, and the state parameter consists of the cloud top altitude $h$, the optical thickness $\tau$, and the particle effective diameter $d_{\text {eff }}$. The cloud is assumed to be $1 \mathrm{~km}$ thick. All other atmospheric properties contribute to the measurement error, implying that the atmospheric state must be provided by another satellite (e.g. AIRS) or reanalysis product [e.g. ERA-Interim; Dee et al., 2011]. Note that for hyperspectral observations, all atmospheric properties can be included in the state parameter [e.g. Merrelli and Turner, 2013].

The optimal estimation framework can be written as follows:

$$
\mathbf{y}=\mathbf{F}\left(\mathbf{x}^{t}, T_{s}^{t}, \epsilon_{\mathbf{s}}^{t}, \mathbf{T}^{t}, \mathbf{R} \mathbf{H}^{t}, H^{t}\right)+\epsilon^{\mathrm{obs}},
$$

where $\mathbf{y}$ is the observation vector, $\mathbf{F}$ the forward model that returns the TOA radiances as a function of all atmospheric information, and $\epsilon^{\mathrm{obs}}$ the observation error. The superscript $t$ indicates the true state, which means that the forward model is assumed perfect. This is consistent with the results of Wang et al. [2016a] who showed that model errors are much smaller than other contributions. Besides the state vector $\mathbf{x}$, the simulated TOA radiance depends on 
surface temperature $T_{s}$, surface spectral emissivity $\epsilon_{\mathbf{s}}$, temperature and relative humidity profiles $\mathbf{T}$ and $\mathbf{R H}$, and ice habit $H$ including actual habit and particle size distribution. Since the true parameters are unknown, the simulated radiance computed from a best estimate of the atmospheric state deviates from the true radiance, so that Eq. 1 can be rewritten as

$$
\begin{aligned}
& \mathbf{y}=\mathbf{F}\left(\mathbf{x}^{t}, T_{s}, \epsilon_{s}, \mathbf{T}, \mathbf{R H}, H\right)+\epsilon^{T_{s}}+\epsilon^{\epsilon_{\mathrm{s}}}+\epsilon^{\mathbf{T}}+\epsilon^{\mathbf{R H}}+\epsilon^{H}+\epsilon^{\mathrm{obs}} \\
& \mathbf{y}=\mathbf{F}\left(\mathbf{x}^{t}, \mathbf{p}\right)+\epsilon^{y},
\end{aligned}
$$

where the measurement error $\epsilon^{y}$ is the sum of errors $\epsilon^{i}=\frac{\partial \mathbf{F}}{\partial p_{i}}\left(p_{i}-p_{i}^{t}\right), \epsilon^{H}$ and $\epsilon^{\text {obs }}$, and $\mathbf{p}$ the parameter vector including all ancillary parameters $p_{i}$. The optimal estimation method aims at determining the posterior distribution of $\mathbf{x}^{t}$ given an a priori on $\mathbf{x}$ and a set of observations. The quantity to be retrieved is thus $P\left(\mathbf{x}^{t} \mid \mathbf{y}\right)$. According to Bayes' theorem:

$$
P\left(\mathbf{x}^{t} \mid \mathbf{y}\right)=\frac{P\left(\mathbf{y} \mid \mathbf{x}^{t}\right) P\left(\mathbf{x}^{t}\right)}{P(\mathbf{y})} .
$$

For sake of simplicity, it is assumed that all pdf are Gaussian. Although this is a strong assumption, it is very widely used, especially in data assimilation, due to the resulting easiness of implementation. In this case,

$$
\begin{aligned}
\ln \left(P\left(\mathbf{y} \mid \mathbf{x}^{t}\right)\right) & =\left[\mathbf{y}-\mathbf{F}\left(\mathbf{x}^{t}, \mathbf{p}\right)\right]^{\top} \mathbf{S}_{y}^{-1}\left[\mathbf{y}-\mathbf{F}\left(\mathbf{x}^{t}, \mathbf{p}\right)\right]+c_{1} \\
\ln \left(P\left(\mathbf{x}^{t}\right)\right) & =\left[\mathbf{x}^{t}-\mathbf{x}^{a}\right]^{\top} \mathbf{S}_{a}^{-1}\left[\mathbf{x}^{t}-\mathbf{x}^{a}\right]+c_{2},
\end{aligned}
$$

where $\mathbf{S}_{y}$ is the error covariance matrix corresponding to the errors $\epsilon^{y}, \mathbf{x}^{a}$ the a priori state parameter and $\mathbf{S}_{a}$ its associated error covariance, and the subscript $\mathrm{T}$ indicates transposition. $\mathbf{S}_{a}$ is such that the a priori uncertainties on $h$ is $4 \mathrm{~km}$, and $\tau$ and $d_{\mathrm{eff}}$ are known within a factor of 10 and 5, respectively. $c_{1}$ and $c_{2}$ are constants. From Eq. 4:

$$
\ln \left(P\left(\mathbf{x}^{t} \mid \mathbf{y}\right)\right)=\left[\mathbf{y}-\mathbf{F}\left(\mathbf{x}^{t}, \mathbf{p}\right)\right]^{\top} \mathbf{S}_{y}^{-1}\left[\mathbf{y}-\mathbf{F}\left(\mathbf{x}^{t}, \mathbf{p}\right)\right]+\left[\mathbf{x}^{t}-\mathbf{x}^{a}\right]^{\top} \mathbf{S}_{a}^{-1}\left[\mathbf{x}^{t}-\mathbf{x}^{a}\right]+c_{3}
$$

It can be shown [Rodgers, 2000] that the posterior distribution $P\left(\mathbf{x}^{t} \mid \mathbf{y}\right)$ is then defined as a multivariate Gaussian distribution with mean $\mathbf{x}^{p}$ and error covariance matrix $\mathbf{S}_{p}$ such that 


$$
\begin{aligned}
\ln \left(P\left(\mathbf{x}^{t} \mid \mathbf{y}\right)\right) & =\left[\mathbf{x}^{t}-\mathbf{x}^{p}\right]^{\top} \mathbf{S}_{p}^{-1}\left[\mathbf{x}^{t}-\mathbf{x}^{p}\right]+c_{0} \\
\mathbf{S}_{p} & =\left(\mathbf{S}_{a}^{-1}+\mathbf{K}_{x}^{\top} \mathbf{S}_{y}^{-1} \mathbf{K}_{x}\right)^{-1} .
\end{aligned}
$$

The matrix $\mathbf{K}_{x}$ contains the derivatives of the TOA radiance with regards to the state parameters, also called the Jacobians:

$$
\mathbf{K}_{x}=\left[\frac{\partial \mathbf{F}}{\partial h}, \frac{\partial \mathbf{F}}{\partial \tau}, \frac{\partial \mathbf{F}}{\partial d_{\mathrm{eff}}}\right]
$$

The state $x^{\text {opt }}$ that maximizes $P\left(\mathbf{x}^{t} \mid \mathbf{y}\right)$ is the maximum likelihood estimation. For retrieval applications it can be estimated using the Gauss-Newton algorithm [Rodgers, 2000] This iterative method is such that the state $\mathbf{x}_{n+1}$ at step $n+1$ is determined from the state $\mathbf{x}_{n}$ at step $n$ by:

$$
\mathbf{x}_{n+1}=\mathbf{x}^{a}+\left(\mathbf{S}_{a}^{-1}+\mathbf{K}_{n}^{T} \mathbf{S}_{y, n}^{-1} \mathbf{K}_{n}\right)^{-1} \mathbf{K}_{n}^{T} \mathbf{S}_{y, n}^{-1}\left[\mathbf{y}-\mathbf{F}\left(\mathbf{x}_{n}\right)+\mathbf{K}_{n}\left(\mathbf{x}_{n}-\mathbf{x}^{a}\right)\right],
$$

where the Jacobians and error covariances matrices are computed at each step $n$.

\subsubsection{Measurement error contributions}

In this study, 3 independent sources of errors are considered: 1) observation error, 2) uncertainties on the surface and atmospheric states, and 3) uncertainties on crystal habit and particle size distribution. All the error sources are assumed independent and the global error covariance matrix $\mathbf{S}_{y}$ is simply the sum of all the individual contributions. Uncertainties in trace gases concentrations, though potentially critical for channels overlapping $\mathrm{CO}_{2}$ or $\mathrm{O}_{3}$ absorption bands, are not considered here. All radiative transfer calculations are performed with MODTRAN v.5.4 [Berk et al., 2005] using the correlated- $k$ method. Multiple scattering is accounted for using DISORT [Stamnes et al., 1988] with 16 streams. The water vapor continuum is computed from the MT-CKD 2.5 parameterization [Clough et al., 2005]. The spectral surface emissivities are taken from Feldman et al. [2014]. For the Arctic winter case, it is assumed that the surface is snow covered, while for the tropical case, it is ocean water.

For the observation error, the instrument NER is assumed similar in each band, equal to $0.01 \mathrm{~W} \mathrm{~m}^{-2} \mathrm{sr}^{-1}$. This is consistent with the laboratory characterization of the FIRR [ $\mathrm{Li}$ bois et al., 2016a] when averaging the signal over a 2-D array of uncooled microbolometers. 
Although such NER is not yet achieved for a FIR imaging system, it is expected that improved optical and electronical design should allow to meet this target within a few years. The absence of any window on the optical path in space will also be beneficial to the response of the instrument, contrary to ground operation in which the FIRR detector works under vacuum in a sealed package. Observation errors in distinct channels are assumed uncorrelated, so that the error covariance matrix $\mathbf{S}_{\mathrm{obs}}$ is diagonal.

Uncertainties on surface emissivity, surface skin temperature and temperature and humidity profiles, altogether referred as ancillary parameters, also affect the quality of the retrieval. Here we assumed that the surface emissivity uncertainties in each band are uncorrelated and equal to 0.01 . Such errors correspond to the imperfect knowledge of surface emissivity in the FIR, not to a mischaracterization of the surface which would result in larger and correlated errors [Feldman et al., 2014]. This implies that such values hold only when the type of surface is well known. Surface temperature uncertainty is taken as $0.5 \mathrm{~K}$. The errors on the temperature and humidity profiles are $1 \mathrm{~K}$ and $15 \%$ at each level. These errors are vertically correlated in an exponential way such that correlation vanishes at $1 \mathrm{~km}$ distance. Such values, also used by Wang et al. [2016a], are consistent with the accuracy of state-ofthe art reanalysis products or IR sounders atmospheric products. The prior and measurement uncertainties used in the study are summarized in Table 3. The case of larger uncertainties for the atmospheric and surface parameters, which might be more realistic for the Arctic, is discussed in Sect. 4. The derivatives of TOA radiance with respect to the ancillary parameters are computed with MODTRAN using finite differences [e.g. Garand et al., 2001] to convert the above mentioned errors into measurement errors (Eq. 3). These measurement errors depend on cloud characteristics and are thus computed for each individual scene.

Studies dedicated to the MIR generally neglect the impact of particle size distribution because it was shown to induce smaller uncertainties than crystal habit [e.g. Cooper et al., 2006]. Although this is true for the MIR, it is not the case in the FIR where particle size distribution can have an impact similar to crystal habit [Baran, 2007]. As a consequence, it is included in the present error analysis because both habit and size distribution uncertainties can result in changes of TOA radiance close to $5 \%$ in FIR bands. To account for those uncertainties, several simulations were performed for various habits and size distributions. Practically, the nine habits of the database of Yang et al. [2013] are used, with the 3 investigated roughness parameters. Size distributions are assumed to follow a modified gamma distribution [e.g. Petty and Huang, 2011; Merrelli and Turner, 2013] with effective variances 
0.1 or 0.2 . For each cloud scene, 54 simulations are thus performed, and the error covariance matrix $\mathbf{S}_{\text {hab }}$ is computed from these simulations.

\subsubsection{Information content}

The information content of a set of observations is estimated through Shannon information content (SIC), which quantifies the entropy reduction of the state parameter resulting from the observations. Here, SIC is defined as:

$$
\mathrm{SIC}=\frac{1}{2} \ln \left|\mathbf{S}_{a} \mathbf{S}_{p}^{-1}\right|
$$

This quantity can be used to compare the information content of observations taken either with different instruments or with a particular set of channels. For instance it has been used to select the most informative channels of IASI [Rabier et al., 2002], which are currently assimilated in numerical weather forecast models, or to identify the most informative channels of MODIS [Cooper et al., 2006; Wang et al., 2016a]. In addition to SIC, the retrieval uncertainty for each state parameter is defined as the square roots of the diagonal elements of $\mathbf{S}_{p}$.

\section{Results}

\subsection{Jacobians of TOA radiance}

The Jacobians of TOA radiance in terms of the state parameters were computed for both Arctic and tropical atmospheres. They are shown in Fig. 5 as a function of cloud optical thickness. Since the bandwidths were selected to equipartition the amount of energy available in each band for the reference Arctic winter case, the energy partition slightly varies with optical thickness. As a consequence, for large optical depth, the amount of energy available in FIR bands exceeds that available in MIR bands, because the cloud scene becomes colder and Planck emission spectrum is shifted towards the FIR. For the same reason, the energy available in FIR bands is similar for the Arctic and tropical reference cases, because FIR channels probe the higher troposphere, which temperature is similar in the Arctic and tropics. On the contrary, MIR observations are greatly advantaged at low optical depth in the tropics because they are sensitive to the high emission of the warm surface. Fortunately, FIR observations do not suffer the disadvantage of MIR observations in cold regions. More generally, 
the thermal contrast between the surface and the cloud is much larger in the MIR, making MIR observations more sensitive to small changes of optical thickness in warm regions.

The most striking point in Fig. 5 is the high sensitivity of FIR TOA radiance to changes in $d_{\text {eff }}$ for the Arctic case. Indeed, while MIR sensitivity is relatively low, and completely vanishes at optical thickness above 10 , the sensitivity around $23 \mu \mathrm{m}$ is much larger and increases with optical thickness. This intrinsic advantage of the FIR results from the larger sensitivity of cloud single scattering properties to effective diameter highlighted in Fig. 3. The small differences of single scattering albedo are amplified through multiple scattering, so that the advantage increases with optical thickness. When clouds become perfectly opaque to surface radiation, their signature only comes from cloud emission. As in the MIR thick clouds are nearly blackbody emitters, their signature is independent of $d_{\text {eff }}$. In the FIR on the contrary, because clouds have emissivity less than one, their signature still carries information about particle size. This is very similar to clouds characteristics at $3.7 \mu \mathrm{m}$ [Wang et al., 2016a] or in the visible [Nakajima and King, 1990]. For the tropical case, FIR channels are mainly advantaged at large optical thickness. At small optical thickness, the smaller sensitivity of MIR channels to $d_{\text {eff }}$ is compensated by the larger thermal contrast between the surface and the extremely cold cloud. This shows that FIR observations can be extremely useful to observe cloud microphysics at the top of optically thick convective towers for instance [Jensen et al., 1996]. Using FIR channels overall offers a way to overcome the natural limit of MIR algorithms at large optical thickness [Iwabuchi et al., 2016].

\subsection{Error covariance matrices}

The error contributions of ancillary parameters and crystal habit for the two reference cases are shown in Fig. 6. Observation errors are not shown because they are much smaller. For the Arctic case, uncertainties on surface temperature and emissivity have a strong impact because the atmosphere is very transparent. They impact more MIR channels, which are more sensitive to the surface, than FIR channels. FIR channels are mostly affected by water vapor uncertainties above the cloud. Crystal habit induces the largest errors in the weakly absorbing channels of the FIR, because multiple scattering amplifies small differences in single scattering properties. This large uncertainty is nevertheless compensated by the advantageous sensitivity to $d_{\text {eff }}$ displayed in Fig. 5. Crystal habit impact is less in the MIR, because single scattering properties are less dependent to crystal habit. For the tropical cloud, errors on ancillary data do not impact much the global error because surface emission barely 
reaches the top of the atmosphere in such wet conditions, and because errors on temperature and humidity profiles below the cloud are largely attenuated by the cloud. Regarding crystal habit, its absolute impact is larger in the MIR because the amount of radiation in these bands is almost 3 times more than in the FIR (Fig. 5). However, the relative uncertainty in simulated radiances due to crystal habit uncertainties remains much larger in the FIR than in the MIR.

These uncertainties are propagated to the retrieved state of the atmosphere through the optimal estimation method. To estimate the contribution of each error source to the total uncertainty of the retrieved state parameters, the posterior uncertainty is first computed accounting for all the sources, and then when a single source contribution is removed. The contribution of the removed source is defined as the difference. Those contributions are shown in Fig. 7 for both reference cases. It shows that in general observation errors are minor, except for the determination of $d_{\mathrm{eff}}$ in the Arctic. More generally, observations uncertainties are more critical in the Arctic because of the lower signal-to-noise ratio. In the Arctic, $h$ and $\tau$ retrievals mainly depend on ancillary parameters uncertainties while in the tropical case ice habit is critical as well because most of the signal comes from the cloud, not from the atmosphere below.

\subsection{Information content analysis}

The information content of a set of observations is assessed through SIC (Sect. 2.4.3). For a given atmospheric configuration, this quantity is used to select channels containing most information in a decreasing order. The first channel selected is that which contains, alone, most information. The second channel is that of the remaining channels, which added to the first selected, adds up most of the remaining information, and so on. Each time a new channel is added, the total information content increases, and the uncertainties on the state parameters decrease. Figure 8 shows the progressive decrease of the uncertainties as the number of channels used increases, for the two reference cases. In both cases, the first selected channel essentially constrains the optical thickness. Thereafter only cloud top altitude and particle effective diameter are constrained. Interestingly, although the first channel selected is in the MIR, the next two are in the FIR. In the tropical case, channels 2 to 5 are in the FIR. It should also be noted that most information is captured with only 5 channels, the reduction in uncertainty beyond 5 channels being marginal. This is consistent with the findings of Wang et al. [2016a]. Regarding the degree of uncertainty, cloud top altitude can be 
estimated within about $\pm 400 \mathrm{~m}$. Optical thickness and effective diameter can be retrieved within approximately $15 \%$ to $20 \%$ error for the Arctic case, and within less than $10 \%$ error in the tropical case.

These uncertainties illustrate two particular cases. To explore how they change as a function of cloud characteristics, the same method was applied to various combinations of cloud thickness and particle effective diameter. The resulting uncertainties are shown in Fig.9. In general, errors for the Arctic case are larger than for the tropics. The noise level of the instrument being fixed, the signal-to-noise ratio is indeed lower in the Arctic because the emitted radiation from the colder scene is lower. The particle effective diameter retrieval performs best for optical thickness above 1 and for effective diameter up to approximately $150 \mu \mathrm{m}$. This is likely to extend the potential of MIR radiometry that generally performs poorly for optical thickness larger than 5 and effective diameter exceeding $100 \mu \mathrm{m}$. The retrievals of optical thickness are best for intermediate optical thickness $(1.0<\tau<10)$ but are also very efficient in the tropics for optically thin and optically thick clouds. Cloud top altitude is very well constrained, except for very thin clouds. Errors on $d_{\text {eff }}$ are often below $15 \%$, making it possible to distinguish without ambiguity small particles from large particles.

Figure 10 shows the 5 most informative filters for both reference cases, when optical thickness and effective diameter are varied from the reference state. The filters 26.2 and $68.5 \mu \mathrm{m}$ often appear in the first positions, which can be explained by the large amount of information they contain about cloud top and effective diameter. In most situations it remains necessary to use at least one MIR channel because it constrains well the optical thickness in the atmospheric window. Note also that some channels have less importance and are seldom selected. This suggests that removing channels 14.3, 19.2 and $33.3 \mu \mathrm{m}$ would not degrade the performance of the radiometer. Such considerations should be borne in mind when designing a new instrument.

\section{Discussion}

\subsection{Added value of FIR observations}

It was shown that FIR observations are most useful when the thermal contrast between the cloud and the surface is low, when the cloud is opaque in the MIR, and when the atmosphere above the cloud is sufficiently transparent to let cloud emission escape to TOA. The 
former conditions are encountered when cloud optical thickness is large, or when the surface is cold. This makes FIR observations very valuable to probe polar ice clouds, and optically thick high altitude ice clouds in general. Although the potential of FIR radiometry has been shown in terms of absolute retrieval performances (Fig. 9), it is worth quantifying the added value relative to MIR only observations. To this end, the method detailed in Sect. 3.3 was applied to a synthetic radiometer made of only the first 4 MIR bands of the 10-band synthetic radiometer. The relative decrease in $d_{\text {eff }}$ uncertainty resulting from the addition of the 6 FIR channels is presented in Fig. 11. As expected, in both Arctic and tropical cases the largest improvement is for optical thickness beyond 10, because those are the conditions where FIR observations are most sensitive to $d_{\text {eff }}$, contrasting with the inherent limitations of MIR observations in such cases. In those cases, the uncertainty is reduced by more than $80 \%$. The improvement is very significant for intermediate optical thickness $(\tau=0.5-5)$ as well, often exceeding $50 \%$. This is particularly true for the Arctic case which is inherently disadvantaged with MIR alone due to the low radiance emitted by the cold surface. At small optical thickness, the uncertainty is barely reduced for the Arctic case. On the contrary, it is largely reduced in the tropical case because the thermal contrast between the surface and the cloud is larger, and the cloud emission in the FIR is unaltered by water vapor absorption above. This highlights the strong potential of FIR observations for studying optically very thin cirrus clouds, especially for large ice crystals $\left(d_{\mathrm{eff}}>50 \mu \mathrm{m}\right)$. To some extent, using FIR observations adds a new retrieval capability for thick clouds and large particles in cases without reflected solar radiation.

Adding FIR channels not only reduces the uncertainties of the retrieved cloud properties, it also reduces the correlations between the retrieved state variables. Table 4 shows these correlations $\rho$ for both reference cases, when only MIR channels are used, and when all channels are used. Adding FIR channels reduces all the correlated errors and is most useful to discriminate between $h$ and $d_{\mathrm{eff}}$.

So far, the obtained results are theoretical because the instrument which they refer to does not exist yet. To give it a more realistic flavor, the same method was applied to the real instruments described in Section 2.2. For this, the added value of the future TICFIRE satellite was quantified with regards to MODIS (see Sect. 2.2). Figure 12 is similar to Fig. 11 except that the reference corresponds to the 10 bands of MODIS, and the full instrument corresponds to MODIS channels complemented by the 9 channels of TICFIRE. The patterns for the particle effective diameter are very similar to those of Fig. 11. The values are slightly 
lower, though, because the 10 channels of MODIS contain more information than the 4 MIR channels of the synthetic radiometer. The largest difference is the more limited improvement for cloud particles beyond $100 \mu \mathrm{m}$ in the tropical case. The reductions in uncertainty for optical thickness and cloud top altitude are also shown for the Arctic case. FIR observations help constrain all cloud parameters for a large variety of cloud characteristics. They also reduce the correlated errors of the retrieved parameters (Table 4). The reductions in uncertainty for cloud top altitude and optical thickness in the tropical case are not shown since they are not as significant as in the Arctic case. These results overall highlight that adding TICFIRE observations to existing MODIS observations would greatly increase the performance of cloud properties retrieval algorithms, in the polar regions but also for thin cirrus and convective ice clouds in the tropics [Hong et al., 2007].

The added value of FIR observations is further illustrated in Fig. 13 (similar to Fig. 8), showing how using TICFIRE observations reduces the uncertainties on cloud properties for a thin and a thick Arctic cloud. It suggests that adding 2 or 3 FIR channels to MODIS would drastically improve cloud retrieval performance. Practically, this could be done at reasonable cost by adding such a FIR radiometer on the A-Train or EarthCARE orbit [Illingworth et al., 2015]. Alternatively, it suggests that a compact radiometer such as TICFIRE, which covers the MIR and the FIR, could entirely replace MODIS for ice cloud monitoring.

\subsection{Range of validity of the algorithm}

All previous results are bound to the assumed uncertainties of the ancillary parameters. Those were chosen to be consistent with the work of Wang et al. [2016a], but may be optimistic, especially for the polar regions where a priori atmospheric profiles are generally poorly constrained. To estimate the sensitivity of the results to the ancillary parameters uncertainties, each individual uncertainty was varied individually, all other things being constant. The impact of such variations on the posterior uncertainties for both reference cases are shown in Fig. 15. For the Arctic case, $h$ retrieval is mostly sensitive to the temperature profile and $\tau$ retrieval also depends on the surface temperature. The posterior error of $d_{\mathrm{eff}}$ is very sensitive to errors on surface emissivity, because FIR channels that mostly constrain $d_{\text {eff }}$ also see the surface. This highlights that surface properties should be well determined to operate the algorithm. It is critical in the Arctic when open leads are present in the scene but ignored by the algorithm, since water and snow have very distinct emissivities in the FIR [Feldman et al., 2014]. The impact of such surface heterogeneities, though crucial, is not fur- 
ther investigated here. In the tropics, all error sources have a similar impact on the retrievals, but the sensitivity is much less than in the Arctic, mostly because the cloud is high and the surface contributon to the TOA radiance limited due to atmospheric absorption below the cloud. These results show that the algorithm remains efficient even when the ancillary parameters are poorly constrained. In addition to this sensitivity study, Figure 14 shows the uncertainty reduction due to the addtion of TICFIRE channels to MODIS channels for large uncertainties on the surface temperature, surface emissivities, and thermodynamical profiles, in agreement with the values chosen by Wang et al. [2016a] (Table 3). The added value of FIR channels is generally reduced compared to the case with better constrained ancillary parameters, but the uncertainty reduction on $d_{\text {eff }}$ slightly increases in the tropics.

The added value of FIR observations for the retrieval of ice cloud properties has been demonstrated for ideal cloud geometries, but it is worth discussing the impact of cloud heterogeneities on retrieval performances. A FIR radiometer is likely to have a spatial footprint much larger than current MIR radiometers. Although the resolution of the TICFIRE instrument is not known so far (it will depend on the field of view, the number of pixels of the detector, the number of filters, the acquisition rate etc.), it should be around $10 \mathrm{~km}$. In comparison, MODIS footprint is around $1 \mathrm{~km}$. As a consequence, the comparison of MIR and FIR information content should properly account for these differences in spatial resolution. Since this is beyond the scope of the present paper, the current results are most valid for large scale homogeneous cloud systems. Such systems, as observed in the Arctic polar night [Grenier et al., 2009], are the main target of TICFIRE at synoptic scales.

The impact of cloud heterogeneity was nevertheless estimated in a simple way, by treating cloud fraction as an ancillary parameter. Fig. 16 shows the error covariance matrices associated to an actual cloud fraction of 0.95 instead of 1 . They are compared to the other error contributions. In the Arctic, assuming that a pixel is entirely filled with clouds when it contains $5 \%$ of clear sky results in errors similar to the uncertainties associated to the temperature profile. In the tropics, small errors on cloud fraction are very critical in the MIR due to the strong thermal contrast between the surface and the cloud. Generally, it means that for cloud fraction less than 0.9 , assuming the pixel is overcast might be the largest source of error of the retrieval. Practically, an operational algorithm should rely on independent sources for cloud cover. 
All these uncertainties were obtained in the case of a homogeneous single layer cloud. Multi-layer cloud systems or subpixel horizontal heterogeneities, which are not accounted for by our 1-D radative transfer computations, are likely to degrade the quality of the retrieval [Fauchez et al., 2015; Wang et al., 2016b]. Accounting for 3D effects in general in remote sensing is a challenging issue, but essential to improve current algorithms [e.g. Schäfer et al., 2016]. The potential of FIR radiometry for studying multi-layer systems should be explored. The spectral variations of water vapor absorption in the FIR are such that some channels may see only the highest clouds while others would sense the whole column. Likewise, FIR could be valuable to study the vertical structure of clouds, which is essential to better understand ice cloud microphysics.

\subsection{Sensitivity to instrument radiometric resolution}

The previous results were obtained assuming a NER of $0.01 \mathrm{~W} \mathrm{~m}^{-2} \mathrm{sr}^{-1}$ for the FIR synthetic radiometer. Although the contribution of the measurement error is minor in the total error budget (Fig. 6), it is worth investigating its impact on the retrieval performance and could provide quantitative targets to the industry in view of meeting scientific requirements requested by the geophysical community. Figure 17 shows the variations of the uncertainties for both reference cases as a function of instrument radiometric resolution. It demonstrates that the retrieval uncertainties in the Arctic case significantly increase when the radiometric resolution exceeds $0.02 \mathrm{~W} \mathrm{~m}^{-2} \mathrm{sr}^{-1}$. On the contrary, upgrading the resolution to $0.001 \mathrm{~W} \mathrm{~m}^{-2} \mathrm{sr}^{-1}$ does not significantly reduce the retrieval uncertainties. The tropical case is less sensitive to sensor noise because the signal-to-noise ratio is favorable in a warmer atmosphere. This suggests that the selected resolution of $0.01 \mathrm{~W} \mathrm{~m}^{-2} \mathrm{sr}^{-1}$ provides a good trade off between realistically achievable technology and retrieval performance. A radiometric resolution worst than $0.03 \mathrm{~W} \mathrm{~m}^{-2} \mathrm{sr}^{-1}$ would be damageable for the quality of the retrieval and efforts are highly recommended to exceed this target.

\subsection{Sensitivity to the number of channels}

The results presented so far were mostly based on the 10-band configuration of the synthetic FIR radiometer. Although it suggests that approximately 5 bands contain the bulk of the information, it is worth exploring the dependence of the performance on the total number of bands of the radiometer. Using thinner bands, for instance, may help select the regions of the spectrum where sensitivity is maximal and where uncertainties due to ancillary 
parameters are minimal. Too narrow bands may however suffer a low signal-to-noise ratio Figure 18a shows how SIC varies with an increasing number of contiguous bands. The increase is significant up to approximately 7 channels, but limited for additional channels. The small oscillations observed beyond 5 channels suggest that an optimal configuration could be degraded by increasing the number of bands due to a spectral shift of those optimal bands. Practically, it suggests that an instrument with around 7 channels would be sufficient. The gain in information content resulting from increasing the number of channels would certainly come at the expanse of acquisition rate, effective spatial resolution, technical complexity and cost, which has to be considered beforehand. Such statement is worthy in view of designing a FIR radiometer dedicated to ice cloud observation.

These results also imply that instruments with a large number of bands could be optimized by merging adjacent bands in order to increase the signal-to-noise ratio. Such considerations could be applied in the case of hyperspectral measurements. Instead of using a limited number of channels for data assimilation [e.g. Rabier et al., 2002], merging channels could for instance result in improved performances without increasing computing time since the effective number of assimilated observations would remain constant. More generally, this points out that instruments dedicated to clouds observation do not require very high spectral resolution. Most recent FIR satellite projects focused on such high spectral resolution, though, because they also aim at providing information about atmospheric profiles and trace gases [e.g. Merrelli and Turner, 2012].

Although 4 to 5 channels are generally sufficient to provide most of the information, these very channels depend on the scene [Cooper et al., 2006]. Here the most useful channels in the 10-band configuration are chosen as follows. For each $\left(\tau, d_{\mathrm{eff}}\right)$ configuration illustrated in Fig. 9, a filter is assigned its rank of selection. Then the sum of all these values is computed for each filter and those with the minimal values are the most informative. For the Arctic winter cases, the 4 most valuable channels are (in decreasing order of importance) $12.3,7.7,68.5$ and $26.2 \mu \mathrm{m}$. For the tropical cases those are the 26.2, 68.5, 33.3 and $10.4 \mu \mathrm{m}$. For the combination of both atmospheres, the most informative channels are the $68.5,26.2,12.3$ and $7.7 \mu \mathrm{m}$. This highlights the necessity of using FIR channels.

From the previous analysis, the number of useful bands was estimated to be less than 10. Since these actual bands were defined only on the basis of equienergetic partition (Sect. 2.2), we now investigate what would be the ideal set of channels for a given number of bands. For 
this, the channels edges are varied from the reference equienergetic configuration. A minimization tool (SciPy [Jones et al., 2001] fmin function) is used to find the configuration that maximizes the information content for each number of bands. This configuration is obtained after 300 iterations of the minimization algorithm. The dashed lines in Fig. 18a show the information content as a function of band numbers for these optimized channels. Although there is no guarantee that the obtained optimized bands correspond to a global optimum, it highlights that a significant amount of information can be gained by choosing appropriately the positions and bandwidths of each band, once the number of channels has been imposed. For instance, using 6 optimized channels for the tropical case is equivalent to using 11 equienergetic channels. These optimized channels are shown in Fig. 18b for the reference cases. It demonstrates that choosing overlapping channels can improve performances, as excluding parts of the spectrum can do by removing segments of the spectrum responsible for increasing uncertainties. These conclusions hold for systems where the amount of available energy in one band is independent of the other bands, as is the case for filter-wheel systems acquiring sequentially in time the different bands. In a system where all spectral channels are acquired simultaneously, though, overlapping would imply that energy is split in multiple bands, which would reduce the signal-to-noise ratio and potentially degrade the overall information content.

\subsection{Retrieval performance}

The present study only focused on the information content of the radiance measurements, without explicitly performing cloud properties retrievals. As in the studies of Cooper et al. [2006] and Merrelli and Turner [2013], the uncertainties obtained for cloud parameters are thus theoretical and bound to the assumptions underlying the optimal estimation method, in particular that all considered errors are unbiased and Gaussian. Practically, departure from this ideal behavior can result in bias that the present theory does not account for [Wang et al., 2016b]. Only the computation of retrievals can assess the actual performance of the method. Although this is beyond the scope of this paper, a retrieval has been applied for the Arctic winter atmosphere, with a representative cloud situated between 5.0 and $6.0 \mathrm{~km}$, of optical thickness 2.7 and effective diameter $76 \mu \mathrm{m}$. For this profile, 20 synthetic FIR radiometer measurements were computed from the true expected measurements, by adding a white Gaussian noise corresponding to a radiometric error of $0.01 \mathrm{~W} \mathrm{~m}^{-2} \mathrm{sr}^{-1}$. For each measurement, the Gauss-Newton algorithm detailed in Section 2.4.1 was applied, starting from an $a$ 
priori $\mathbf{x}^{a}$ that is relatively far from the true state $\left(h=8 \mathrm{~km}, \tau=1.0\right.$ and $\left.d_{\mathrm{eff}}=40 \mu \mathrm{m}\right)$. The iterations are stopped when the difference between two consecutive states or the deviation from the observations is sufficiently small, or when the number of steps reaches 15 .

For this particular case, the average retrieved values of $h, \tau$ and $d_{\text {eff }}$ are $6.0 \mathrm{~km}, 2.6$ and $77 \mu \mathrm{m}$, respectively. The standard deviations among the 10 measurements are $60 \mathrm{~m}$, 0.1 and $9 \mu \mathrm{m}$. This end-to-end simulation highlights the very good performance of the retrieval for this particular example. It is characterized by nearly no bias, and standard deviations even better than the 1-sigma uncertainties expected from the optimal estimation framework, namely $290 \mathrm{~m}, 0.34$ and $12 \mu \mathrm{m}$. The obtained correlated errors $\left(\rho_{\ln \tau, \ln d_{\mathrm{eff}}}=-0.64\right.$, $\rho_{\ln \tau, h}=-0.63$ and $\rho_{\ln d_{\text {eff }}, h}=0.48$ ) are similar to those expected from the optimal estimation $\left(\rho_{\ln \tau, \ln d_{\mathrm{eff}}}=-0.34, \rho_{\ln \tau, h}=-0.71\right.$ and $\left.\rho_{\ln d_{\mathrm{eff}}, h}=0.1\right)$ except for $h$ and $\ln d_{\mathrm{eff}}$. For an exhaustive validation of the algorithm, the retrieval should nevertheless be applied to a variety of atmospheric conditions and cloud altitudes as in Wang et al. [2016b]. To perform such exercise, and in view of the development of an operational retrieval product, a faster radiative transfer code is recommended, such as those presented in Wang et al. [2016a] or [Yi et al., 2016].

\section{Conclusion}

This paper investigated the potential of FIR radiometry for the characterization of ice cloud properties, with a special focus on cloud particle effective diameter. To this end we used the optimal estimation framework, which provides the best estimate of cloud properties and the associated uncertainties given an a priori and a set of radiance observations. It was shown that in the polar regions and the upper tropical troposphere FIR radiances contain much more information about cloud particle effective diameter than MIR radiances. This results from the advantageous spectral shift of Planck emission function in cold conditions, and from the strong sensitivity of cloud particle single scattering properties to effective diameter in the FIR. Using FIR observations could thus overcome the limitations of common MIR-based algorithms, increasing retrievals accuracy by more than $50 \%$ in most cases, and by nearly $100 \%$ in some conditions. This would extend the range of validity of existing algorithms to larger optical thickness, larger effective diameter, and to the polar regions in absence of sunlight, thus offering the possibility to observe the initiation of precipitation. Such advancements are promising for studying the microphysics of convective towers and poorly known polar thin ice clouds, which are known to be sensitive to anthropogenic influ- 
ence through ice nucleation processes. Practically, the performance of existing FIR sensors are such that a radiometer with 2 or 3 FIR channels would be sufficient to significantly complement MIR observations. Increasing sensor radiometric resolution could provide another 2 -fold in precision, and optimizing the channels spectral characteristics appears as another venue to improve retrieval performance. Future work should focus on the assessment of FIRbased retrievals, exploring a larger range of cloud cases. This could be done by coupling a FIR radiometer simulator to outputs of reanalysis or regional climate simulations. In summary, this work highlights the relevance of adding FIR observations to the existing Earth observing system. Such FIR satellite is technologically feasible, and highly recommended. In addition, it could be performed at a reasonable cost compared to projects involving high spectral resolution instruments.

\section{Acknowledgments}

We are grateful to the three anonymous reviewers who provided positive inputs that helped improve the quality of the manuscript. This work was in part supported by the Canadian Space Agency through the FAST program and by NETCARE (Network on Climate and Aerosols: Addressing Key Uncertainties in Remote Canadian Environments, a CCAR (Climate Change and Atmospheric Research) program at NSERC (Natural Sciences and Engineering Research Council). We also thank Institut national d'Optique (INO) for instructive discussions on microbolometers. We are grateful to Ping Yang for providing the ice clouds single scattering database. The two reference atmospheric profiles and the spectral reflectances used for the snow and ocean surfaces are given as supplementary data. The radiative transfer simulations were all performed with MODTRAN v. 5.4 (http://modtran.spectral.com). These simulations and the codes used to generate the figures are available from Quentin $\mathrm{Li}$ bois.

\section{References}

Aumann, H., M. Chahine, C. Gautier, M. Goldberg, E. Kalnay, L. McMillin, H. Revercomb, P. Rosenkranz, W. Smith, D. Staelin, L. Strow, and J. Susskind (2003), AIRS/AMSU/HSB on the aqua mission: design, science objectives, data products, and processing systems, IEEE Transactions on Geoscience and Remote Sensing, 41(2), 253-264, doi: 10.1109/TGRS.2002.808356. 
Austin, R. T., A. J. Heymsfield, and G. L. Stephens (2009), Retrieval of ice cloud microphysical parameters using the CloudSat millimeter-wave radar and temperature, Journal of Geophysical Research, 114, doi:10.1029/2008JD010049.

Baran, A. J. (2007), The impact of cirrus microphysical and macrophysical properties on upwelling far-infrared spectra, Quarterly Journal of the Royal Meteorological Society, 133(627), 1425-1437, doi:10.1002/qj.132.

Baum, B. A., R. F. Arduini, B. A. Wielicki, P. Minnis, and S.-C. Tsay (1994), Multilevel cloud retrieval using multispectral HIRS and AVHRR data: Nighttime oceanic analysis, Journal of Geophysical Research, 99(D3), 5499, doi:10.1029/93JD02856.

Berk, A., G. P. Anderson, P. K. Acharya, L. S. Bernstein, L. Muratov, J. Lee, M. Fox, S. M. Adler-Golden, J. H. Chetwynd, M. L. Hoke, R. B. Lockwood, J. A. Gardner, T. W. Cooley, C. C. Borel, and P. E. Lewis (2005), MODTRAN 5: a reformulated atmospheric band model with auxiliary species and practical multiple scattering options: update, p. 662, doi: $10.1117 / 12.606026$.

Blanchet, J.-P., and E. Girard (1995), Water vapor-temperature feedback in the formation of continental Arctic air: its implication for climate, Science of The Total Environment, 160161, 793-802, doi:10.1016/0048-9697(95)04412-T.

Blanchet, J.-P., A. Royer, F. Châteauneuf, Y. Bouzid, Y. Blanchard, J.-F. Hamel, J. de Lafontaine, P. Gauthier, N. T. O'Neill, O. Pancrati, and L. Garand (2011), TICFIRE: a far infrared payload to monitor the evolution of thin ice clouds, pp. $81,761 \mathrm{~K}-81,761 \mathrm{~K}-11$, doi:10.1117/12.898577.

Blumstein, D., G. Chalon, T. Carlier, C. Buil, P. Hebert, T. Maciaszek, G. Ponce, T. Phulpin, B. Tournier, D. Simeoni, P. Astruc, A. Clauss, G. Kayal, and R. Jegou (2004), IASI instrument: technical overview and measured performances, pp. 196-207, doi:10.1117/12. 560907.

Clough, S., M. Shephard, E. Mlawer, J. Delamere, M. Iacono, K. Cady-Pereira, S. Boukabara, and P. Brown (2005), Atmospheric radiative transfer modeling: a summary of the AER codes, Journal of Quantitative Spectroscopy and Radiative Transfer, 91(2), 233-244, doi:10.1016/j.jqsrt.2004.05.058.

Cooper, S. J., T. S. L'Ecuyer, P. Gabriel, A. J. Baran, and G. L. Stephens (2006), Objective Assessment of the Information Content of Visible and Infrared Radiance Measurements for Cloud Microphysical Property Retrievals over the Global Oceans. Part II: Ice Clouds, Journal of Applied Meteorology and Climatology, 45(1), 42-62, doi:10.1175/JAM2327.1. 
Curry, J. A., J. L. Schramm, M. C. Serreze, and E. E. Ebert (1995), Water vapor feedback over the Arctic Ocean, Journal of Geophysical Research, 100(D7), 14,223, doi:10.1029/ 95JD00824.

Dee, D. P., S. M. Uppala, A. J. Simmons, P. Berrisford, P. Poli, S. Kobayashi, U. Andrae, M. A. Balmaseda, G. Balsamo, P. Bauer, P. Bechtold, A. C. M. Beljaars, L. van de Berg, J. Bidlot, N. Bormann, C. Delsol, R. Dragani, M. Fuentes, A. J. Geer, L. Haimberger, S. B. Healy, H. Hersbach, E. V. Hólm, L. Isaksen, P. Kållberg, M. Köhler, M. Matricardi, A. P. McNally, B. M. Monge-Sanz, J.-J. Morcrette, B.-K. Park, C. Peubey, P. de Rosnay, C. Tavolato, J.-N. Thépaut, and F. Vitart (2011), The ERA-Interim reanalysis: configuration and performance of the data assimilation system, Quarterly Journal of the Royal Meteorological Society, 137(656), 553-597, doi:10.1002/qj.828.

Fauchez, T., P. Dubuisson, C. Cornet, F. Szczap, A. Garnier, J. Pelon, and K. Meyer (2015), Impacts of cloud heterogeneities on cirrus optical properties retrieved from space-based thermal infrared radiometry, Atmospheric Measurement Techniques, 8, 633-647.

Feldman, D. R., W. D. Collins, R. Pincus, X. Huang, and X. Chen (2014), Far-infrared surface emissivity and climate, Proceedings of the National Academy of Sciences, 111(46), $16,297-16,302$.

Garand, L., D. S. Turner, M. Larocque, J. Bates, S. Boukabara, P. Brunel, F. Chevallier, G. Deblonde, R. Engelen, M. Hollingshead, D. Jackson, G. Jedlovec, J. Joiner, T. Kleespies, D. S. McKague, L. McMillin, J.-L. Moncet, J. R. Pardo, P. J. Rayer, E. Salathe, R. Saunders, N. A. Scott, P. Van Delst, and H. Woolf (2001), Radiance and Jacobian intercomparison of radiative transfer models applied to HIRS and AMSU channels, Journal of Geophysical Research: Atmospheres, 106(D20), 24,017-24,031, doi: 10.1029/2000JD000184.

Grenier, P., J. Blanchet, and R. Munoz-Alpizar (2009), Study of polar thin ice clouds and aerosols seen by CloudSat and CALIPSO during midwinter 2007, Journal of Geophysical Research, 114(D9), doi:10.1029/2008JD010927.

Haladay, T., and G. Stephens (2009), Characteristics of tropical thin cirrus clouds deduced from joint CloudSat and CALIPSO observations, Journal of Geophysical Research, 114, doi:10.1029/2008JD010675.

Heli Wei, Ping Yang, Jun Li, B. Baum, Hung-Lung Huang, S. Platnick, Yongxiang Hu, and L. Strow (2004), Retrieval of semitransparent ice cloud optical thickness from atmospheric infrared sounder (AIRS) measurements, IEEE Transactions on Geoscience and Remote 
Sensing, 42(10), 2254-2267, doi:10.1109/TGRS.2004.833780.

Holz, R. E., S. Platnick, K. Meyer, M. Vaughan, A. Heidinger, P. Yang, G. Wind, S. Dutcher, S. Ackerman, N. Amarasinghe, F. Nagle, and C. Wang (2016), Resolving ice cloud optical thickness biases between CALIOP and MODIS using infrared retrievals, Atmospheric Chemistry and Physics, 16(8), 5075-5090, doi:10.5194/acp-16-5075-2016.

Hong, G., P. Yang, B.-C. Gao, B. A. Baum, Y. X. Hu, M. D. King, and S. Platnick (2007), High Cloud Properties from Three Years of MODIS Terra and Aqua Collection-4 Data over the Tropics, Journal of Applied Meteorology and Climatology, 46(11), 1840-1856, doi:10.1175/2007JAMC1583.1.

Illingworth, A. J., H. W. Barker, A. Beljaars, M. Ceccaldi, H. Chepfer, N. Clerbaux, J. Cole, J. Delanoë, C. Domenech, D. P. Donovan, S. Fukuda, M. Hirakata, R. J. Hogan, A. Huenerbein, P. Kollias, T. Kubota, T. Nakajima, T. Y. Nakajima, T. Nishizawa, Y. Ohno, H. Okamoto, R. Oki, K. Sato, M. Satoh, M. W. Shephard, A. Velázquez-Blázquez, U. Wandinger, T. Wehr, and G.-J. van Zadelhoff (2015), The EarthCARE Satellite: The Next Step Forward in Global Measurements of Clouds, Aerosols, Precipitation, and Radiation, Bulletin of the American Meteorological Society, 96(8), 1311-1332, doi: 10.1175/BAMS-D-12-00227.1.

Inoue, T. (1985), On the temperature and effective emissivity determination of semitransparent cirrus clouds by bi-spectral measurements in the $10 \mu \mathrm{m}$ window region, Journal of the Meteorological Society of Japan, 63(1), 88-99.

Iwabuchi, H., S. Yamada, S. Katagiri, P. Yang, and H. Okamoto (2014), Radiative and Microphysical Properties of Cirrus Cloud Inferred from Infrared Measurements Made by the Moderate Resolution Imaging Spectroradiometer (MODIS). Part I: Retrieval Method, Journal of Applied Meteorology and Climatology, 53(5), 1297-1316, doi: 10.1175/JAMC-D-13-0215.1.

Iwabuchi, H., M. Saito, Y. Tokoro, N. S. Putri, and M. Sekiguchi (2016), Retrieval of radiative and microphysical properties of clouds from multispectral infrared measurements, Progress in Earth and Planetary Science, 3(1), doi:10.1186/s40645-016-0108-3.

Jensen, E. J., O. B. Toon, L. Pfister, and H. B. Selkirk (1996), Dehydration of the upper troposphere and lower stratosphere by subvisible cirrus clouds near the tropical tropopause, Geophysical Research Letters, 23(8), 825-828, doi:10.1029/96GL00722.

Jones, E., T. Oliphant, P. Peterson, et al. (2001-), SciPy: Open source scientific tools for Python. 
Kayetha, V. K., and R. L. Collins (2016), Optically thin midlevel ice clouds derived from Cloud Aerosol Lidar and Infrared Pathfinder Satellite Observations, Journal of Applied Remote Sensing, 10(4), 046,007, doi:10.1117/1.JRS.10.046007.

King, M., Y. Kaufman, W. Menzel, and D. Tanre (1992), Remote sensing of cloud, aerosol, and water vapor properties from the moderate resolution imaging spectrometer (MODIS), IEEE Transactions on Geoscience and Remote Sensing, 30(1), 2-27, doi:10.1109/36. 124212.

King, M. D., S. Platnick, P. Yang, G. T. Arnold, M. A. Gray, J. C. Riedi, S. A. Ackerman, and K.-N. Liou (2004), Remote Sensing of Liquid Water and Ice Cloud Optical Thickness and Effective Radius in the Arctic: Application of Airborne Multispectral MAS Data, Journal of Atmospheric and Oceanic Technology, 21(6), 857-875, doi: 10.1175/1520-0426(2004)021<0857:RSOLWA>2.0.CO;2.

L'Ecuyer, T. S., P. Gabriel, K. Leesman, S. J. Cooper, and G. L. Stephens (2006), Objective Assessment of the Information Content of Visible and Infrared Radiance Measurements for Cloud Microphysical Property Retrievals over the Global Oceans. Part I: Liquid Clouds, Journal of Applied Meteorology and Climatology, 45(1), 20-41, doi: 10.1175/JAM2326.1.

Libois, Q., C. Proulx, L. Ivanescu, L. Coursol, L. S. Pelletier, Y. Bouzid, F. Barbero, E. Girard, and J.-P. Blanchet (2016a), A microbolometer-based far infrared radiometer to study thin ice clouds in the Arctic, Atmospheric Measurement Techniques, 9(4), 1817-1832, doi: 10.5194/amt-9-1817-2016.

Libois, Q., L. Ivanescu, J.-P. Blanchet, H. Schulz, H. Bozem, W. R. Leaitch, J. Burkart, J. P. Abbatt, A. B. Herber, A. A. Aliabadi, and E. Girard (2016b), Airborne observations of far-infrared upwelling radiance in the arctic, Atmospheric Chemistry and Physics, 16(24), $15,689-15,707$.

Liu, Y., S. A. Ackerman, B. C. Maddux, J. R. Key, and R. A. Frey (2010), Errors in Cloud Detection over the Arctic Using a Satellite Imager and Implications for Observing Feedback Mechanisms, Journal of Climate, 23(7), 1894-1907, doi:10.1175/2009JCLI3386.1.

McCleese, D. J., J. T. Schofield, F. W. Taylor, S. B. Calcutt, M. C. Foote, D. M. Kass, C. B. Leovy, D. A. Paige, P. L. Read, and R. W. Zurek (2007), Mars Climate Sounder: An investigation of thermal and water vapor structure, dust and condensate distributions in the atmosphere, and energy balance of the polar regions, Journal of Geophysical Research, 112(E5), doi:10.1029/2006JE002790. 
Merrelli, A. (2012), The atmospheric information content of earth's far infrared spectrum.

Merrelli, A., and D. D. Turner (2012), Comparing information content of upwelling farinfrared and midinfrared radiance spectra for clear atmosphere profiling, Journal of Atmospheric and Oceanic Technology, 29(4), 510-526.

Merrelli, A., and D. D. Turner (2013), Information content for cloud ice microphysics in the FIR radiance spectrum, pp. 348-351, doi:10.1063/1.4804778.

Nakajima, T., and M. D. King (1990), Determination of the Optical Thickness and Effective Particle Radius of Clouds from Reflected Solar Radiation Measurements. Part I: Theory, Journal of the Atmospheric Sciences, 47(15), 1878-1893, doi:10.1175/1520-0469(1990) 047<1878:DOTOTA>2.0.CO;2.

Ngo Phong, L., C. Proulx, H. Oulachgar, and F. Châteauneuf (2015), Far infrared microbolometers for radiometric measurements of ice cloud, p. 93750G, doi:10.1117/12. 2076219.

Palchetti, L., C. Belotti, G. Bianchini, F. Castagnoli, B. Carli, U. Cortesi, M. Pellegrini, C. Camy-Peyret, P. Jeseck, and Y. Té (2006), Technical note: First spectral measurement of the Earth's upwelling emission using an uncooled wideband Fourier transform spectrometer, Atmospheric Chemistry and Physics, 6(12), 5025-5030, doi:10.5194/ acp-6-5025-2006.

Palchetti, L., G. Di Natale, and G. Bianchini (2016a), Remote sensing of cirrus cloud microphysical properties using spectral measurements over the full range of their thermal emission: CIRRUS CLOUD FAR IR SIGNATURE, Journal of Geophysical Research: Atmospheres, 12l(18), 10,804-10,819, doi:10.1002/2016JD025162.

Palchetti, L., M. Olivieri, C. Pompei, D. Labate, H. Brindley, G. Di Natale, and G. Bianchini (2016b), The Far Infrared FTS for the FORUM Mission, p. FTu3C.1, OSA, doi:10.1364/ FTS.2016.FTu3C.1.

Petty, G. W., and W. Huang (2011), The Modified Gamma Size Distribution Applied to Inhomogeneous and Nonspherical Particles: Key Relationships and Conversions, Journal of the Atmospheric Sciences, 68(7), 1460-1473, doi:10.1175/2011JAS3645.1.

Platnick, S., M. King, S. Ackerman, W. Menzel, B. Baum, J. Riedi, and R. Frey (2003), The MODIS cloud products: algorithms and examples from terra, IEEE Transactions on Geoscience and Remote Sensing, 41(2), 459-473, doi:10.1109/TGRS.2002.808301.

Rabier, F., N. Fourrié, D. Chafaï, and P. Prunet (2002), Channel selection methods for Infrared Atmospheric Sounding Interferometer radiances, Quarterly Journal of the Royal 
Meteorological Society, 128(581), 1011-1027, doi:10.1256/0035900021643638.

Rodgers, C. D. (2000), Inverse methods for atmospheric sounding: theory and practice, vol. 2, World scientific.

Schäfer, S. A., R. J. Hogan, C. Klinger, J. C. Chiu, and B. Mayer (2016), Representing 3-d cloud radiation effects in two-stream schemes: 1 . longwave considerations and effective cloud edge length, Journal of Geophysical Research: Atmospheres, 121(14), 8567-8582.

Shi, S.-C., S. Paine, Q.-J. Yao, Z.-H. Lin, X.-X. Li, W.-Y. Duan, H. Matsuo, Q. Zhang, J. Yang, M. Ashley, et al. (2016), Terahertz and far-infrared windows opened at Dome A in Antarctica, Nature Astronomy, 1, 0001.

Spankuch, D., and W. Döhler (1985), Radiative properties of cirrus clouds in the middle IR derived from Fourier spectrometer measurements from space, Zeitschrift für Meteorologie, 35(6), 314-324.

Stamnes, K., S.-C. Tsay, W. Wiscombe, and K. Jayaweera (1988), Numerically stable algorithm for discrete-ordinate-method radiative transfer in multiple scattering and emitting layered media, Applied Optics, 27(12), 2502-2509, doi:10.1364/AO.27.002502.

Stephens, G. L., D. G. Vane, R. J. Boain, G. G. Mace, K. Sassen, Z. Wang, A. J. Illingworth, E. J. O'Connor, W. B. Rossow, S. L. Durden, S. D. Miller, R. T. Austin, A. Benedetti, C. Mitrescu, and T. CloudSat Science Team (2002), THE CLOUDSAT MISSION AND THE A-TRAIN: A New Dimension of Space-Based Observations of Clouds and Precipitation, Bulletin of the American Meteorological Society, 83(12), 1771-1790, doi: 10.1175/BAMS-83-12-1771.

Turner, D. D., and E. J. Mlawer (2010), The Radiative Heating in Underexplored Bands Campaigns, Bulletin of the American Meteorological Society, 91(7), 911-923, doi: 10.1175/2010BAMS2904.1.

Wang, C., P. Yang, B. A. Baum, S. Platnick, A. K. Heidinger, Y. Hu, and R. E. Holz (2011), Retrieval of Ice Cloud Optical Thickness and Effective Particle Size Using a Fast Infrared Radiative Transfer Model, Journal of Applied Meteorology and Climatology, 50(11), 2283-2297, doi:10.1175/JAMC-D-11-067.1.

Wang, C., P. Yang, S. Platnick, A. K. Heidinger, B. A. Baum, T. Greenwald, Z. Zhang, and R. E. Holz (2013), Retrieval of Ice Cloud Properties from AIRS and MODIS Observations Based on a Fast High-Spectral-Resolution Radiative Transfer Model, Journal of Applied Meteorology and Climatology, 52(3), 710-726, doi:10.1175/JAMC-D-12-020.1. 
Wang, C., S. Platnick, Z. Zhang, K. Meyer, and P. Yang (2016a), Retrieval of ice cloud properties using an optimal estimation algorithm and MODIS infrared observations: 1 . Forward model, error analysis, and information content: IR-Based Ice Cloud Retrieval Algorithm, Journal of Geophysical Research: Atmospheres, 121(10), 5809-5826, doi: $10.1002 / 2015 J D 024526$.

Wang, C., S. Platnick, Z. Zhang, K. Meyer, G. Wind, and P. Yang (2016b), Retrieval of ice cloud properties using an optimal estimation algorithm and MODIS infrared observations: 2. Retrieval evaluation: Comparison of Ice Cloud Retrievals, Journal of Geophysical Research: Atmospheres, 121(10), 5827-5845, doi:10.1002/2015JD024528.

Wang, X., and J. R. Key (2005), Arctic Surface, Cloud, and Radiation Properties Based on the AVHRR Polar Pathfinder Dataset. Part I: Spatial and Temporal Characteristics, Journal of Climate, 18(14), 2558-2574, doi:10.1175/JCLI3438.1.

Wielicki, B. A., D. F. Young, M. G. Mlynczak, K. J. Thome, S. Leroy, J. Corliss, J. G. Anderson, C. O. Ao, R. Bantges, F. Best, K. Bowman, H. Brindley, J. J. Butler, W. Collins, J. A. Dykema, D. R. Doelling, D. R. Feldman, N. Fox, X. Huang, R. Holz, Y. Huang, Z. Jin, D. Jennings, D. G. Johnson, K. Jucks, S. Kato, D. B. Kirk-Davidoff, R. Knuteson, G. Kopp, D. P. Kratz, X. Liu, C. Lukashin, A. J. Mannucci, N. Phojanamongkolkij, P. Pilewskie, V. Ramaswamy, H. Revercomb, J. Rice, Y. Roberts, C. M. Roithmayr, F. Rose, S. Sandford, E. L. Shirley, W. L. Smith, B. Soden, P. W. Speth, W. Sun, P. C. Taylor, D. Tobin, and X. Xiong (2013), Achieving Climate Change Absolute Accuracy in Orbit, Bulletin of the American Meteorological Society, 94(10), 1519-1539, doi: 10.1175/BAMS-D-12-00149.1.

Winker, D. M., J. R. Pelon, and M. P. McCormick (2003), The CALIPSO mission: spaceborne lidar for observation of aerosols and clouds, pp. 1-11, doi:10.1117/12.466539.

Xiong, X., B. Wenny, Aisheng Wu, W. Barnes, and V. Salomonson (2009), Aqua MODIS Thermal Emissive Band On-Orbit Calibration, Characterization, and Performance, IEEE Transactions on Geoscience and Remote Sensing, 47(3), 803-814, doi:10.1109/TGRS. 2008.2005109.

Yang, P., M. G. Mlynczak, H. Wei, D. P. Kratz, B. A. Baum, Y. X. Hu, W. J. Wiscombe, A. Heidinger, and M. I. Mishchenko (2003), Spectral signature of ice clouds in the farinfrared region: Single-scattering calculations and radiative sensitivity study, Journal of Geophysical Research: Atmospheres, 108(D18). 
Table 1. Cloud characteristics for the two reference cases.

\begin{tabular}{ccc}
\hline Quantity & Arctic winter & Tropics \\
\hline$\tau$ & 1.0 & 1.0 \\
$d_{\text {eff }}$ & $60 \mu \mathrm{m}$ & $60 \mu \mathrm{m}$ \\
$h_{\text {bot }}$ & $5 \mathrm{~km}$ & $14 \mathrm{~km}$ \\
$h_{\text {top }}$ & $6 \mathrm{~km}$ & $15 \mathrm{~km}$ \\
\hline
\end{tabular}

Yang, P., L. Bi, B. A. Baum, K.-N. Liou, G. W. Kattawar, M. I. Mishchenko, and B. Cole (2013), Spectrally Consistent Scattering, Absorption, and Polarization Properties of Atmospheric Ice Crystals at Wavelengths from 0.2 to $100 \mu \mathrm{m}$, Journal of the Atmospheric Sciences, 70(1), 330-347, doi:10.1175/JAS-D-12-039.1.

Yi, B., P. Yang, Q. Liu, P. van Delst, S.-A. Boukabara, and F. Weng (2016), Improvements on the ice cloud modeling capabilities of the Community Radiative Transfer Model: Improved Ice Optical Properties of CRTM, Journal of Geophysical Research: Atmospheres, 121(22), 13,577-13,590, doi:10.1002/2016JD025207.

Yue, Q., and K. N. Liou (2009), Cirrus cloud optical and microphysical properties determined from AIRS infrared spectra, Geophysical Research Letters, 36(5), doi:10.1029/ 2008 GL036502. 
Table 2. Spectral bands of MODIS and TICFIRE used in this study. The values indicate the central wavelengths in $\mu \mathrm{m}$.

\begin{tabular}{ccc}
\hline Band number & MODIS & TICFIRE \\
\hline 1 & 6.8 & 8.7 \\
2 & 7.3 & 11.0 \\
3 & 8.6 & 13.0 \\
4 & 9.7 & 17.8 \\
5 & 11.0 & 18.5 \\
6 & 12.0 & 19.5 \\
7 & 13.4 & 21.5 \\
8 & 13.7 & 25 \\
9 & 13.9 & 40 \\
10 & 14.2 & - \\
\hline
\end{tabular}

Table 3. Uncertainties for the prior state parameters, for the ancillary parameters and for the radiance measurements used throughout the study. The high values are used in Section 4.2.

\begin{tabular}{c|cccccccc} 
Quantity & $\ln \tau$ & $\ln d_{\mathrm{eff}}$ & $h$ & $T_{S}$ & $\epsilon_{S}$ & $T$ & $\mathrm{RH}$ & Radiance \\
\hline Uncertainty (nominal) & $\ln 10$ & $\ln 5$ & $4 \mathrm{~km}$ & $0.5 \mathrm{~K}$ & 0.01 & $1 \mathrm{~K}$ & $15 \%$ & $0.01 \mathrm{~W} \mathrm{~m}^{-2}$ \\
Uncertainty (high) & - & - & - & $2 \mathrm{~K}$ & 0.03 & $2 \mathrm{~K}$ & $25 \%$ & -
\end{tabular}

Table 4. Correlations between the retrieved state parameters for the two reference cases, when MIR only or MIR and FIR channels are used. The quantities in parenthesis indicate the correlated errors when MODIS only or MODIS and TICFIRE channels are used.

\begin{tabular}{c|cc|cc} 
& Arctic winter & \multicolumn{2}{|c}{ Tropics } \\
\cline { 2 - 5 } & MIR & MIR + FIR & MIR & MIR + FIR \\
\hline$\rho_{\ln \tau, \ln d_{\mathrm{eff}}}$ & $-0.88(-0.65)$ & $-0.13(-0.02)$ & $-0.56(-0.43)$ & $-0.41(-0.56)$ \\
$\rho_{\ln \tau, h}$ & $-0.97(-0.97)$ & $-0.67(-0.49)$ & $-0.87(-0.60)$ & $-0.31(-0.15)$ \\
$\rho_{\ln d_{\mathrm{eff}}, h}$ & $0.87(0.67)$ & $-0.016(0.06)$ & $0.54(0.30)$ & $-0.20(-0.06)$
\end{tabular}




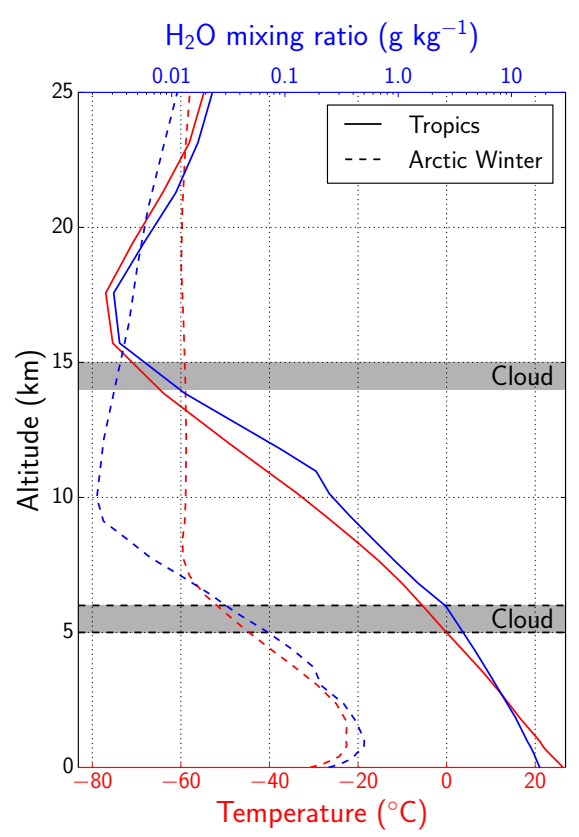

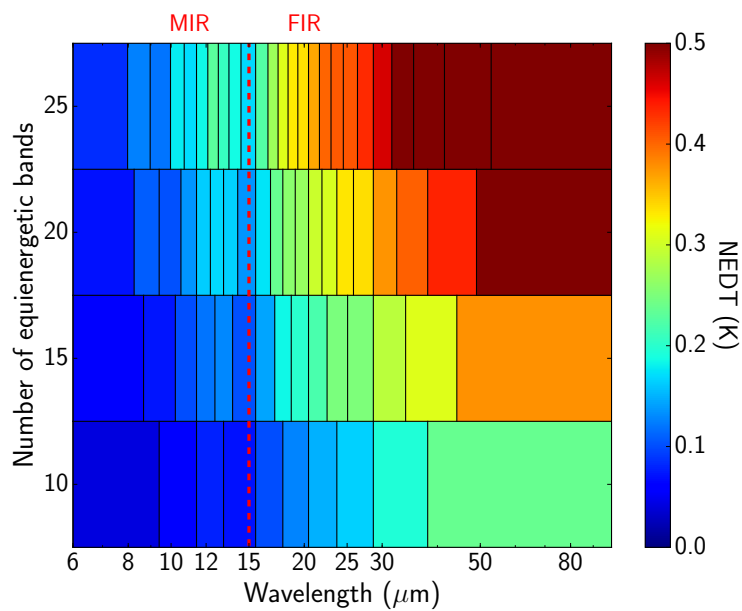

Figure 2. NEDT in each spectral channel as a function of total number of equienergetic bands, for a blackbody at $250 \mathrm{~K}$. The NER is assumed spectrally constant and equals $0.01 \mathrm{~W} \mathrm{~m}^{-2} \mathrm{sr}^{-1}$. 

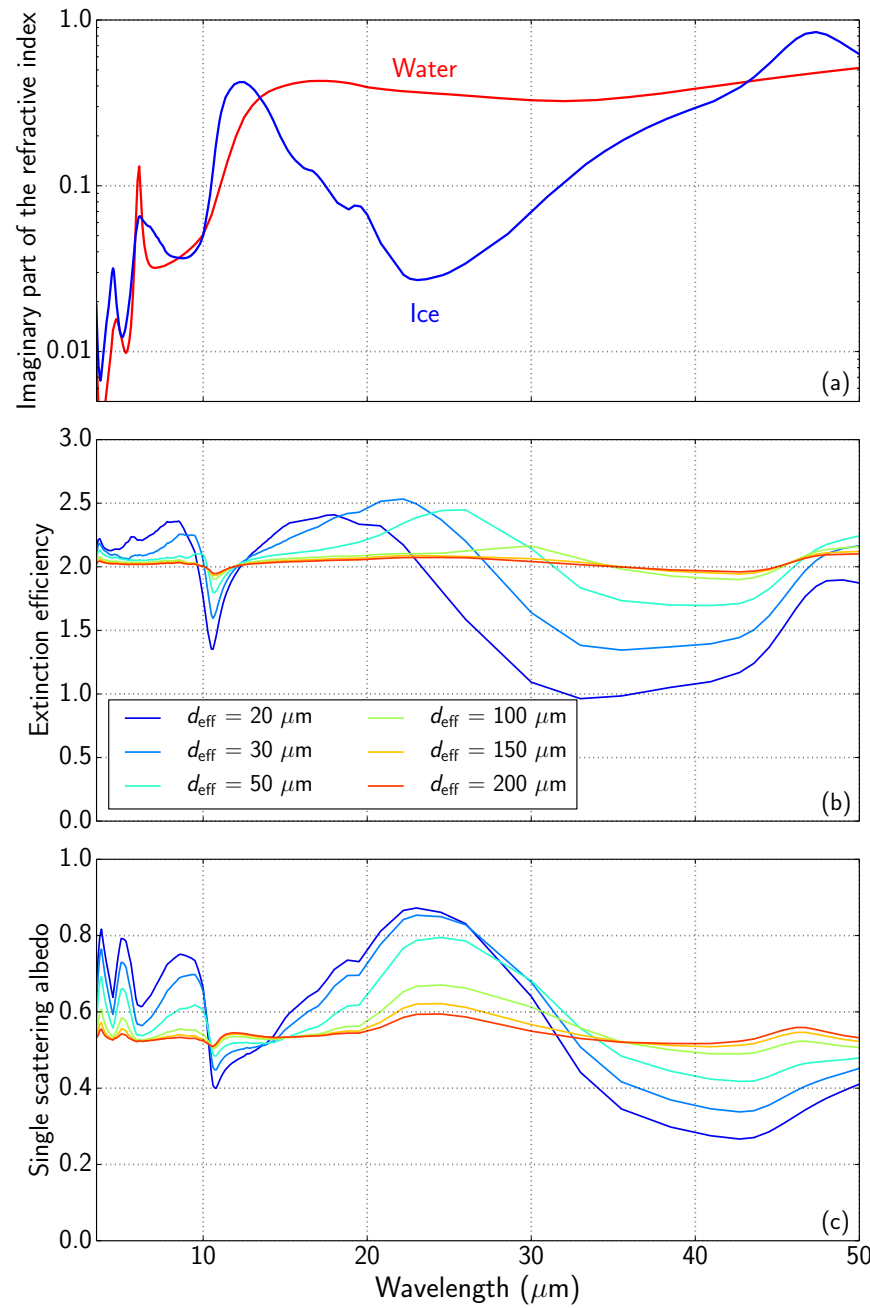

Figure 3. (a) Imaginary part of ice and water refractive indices in the range $3.5-50 \mu \mathrm{m}$. Spectral variations of the (b) extinction coefficient and (c) single scattering albedo for a distribution of severely roughened column aggregates from the database of Yang et al. [2013]. 

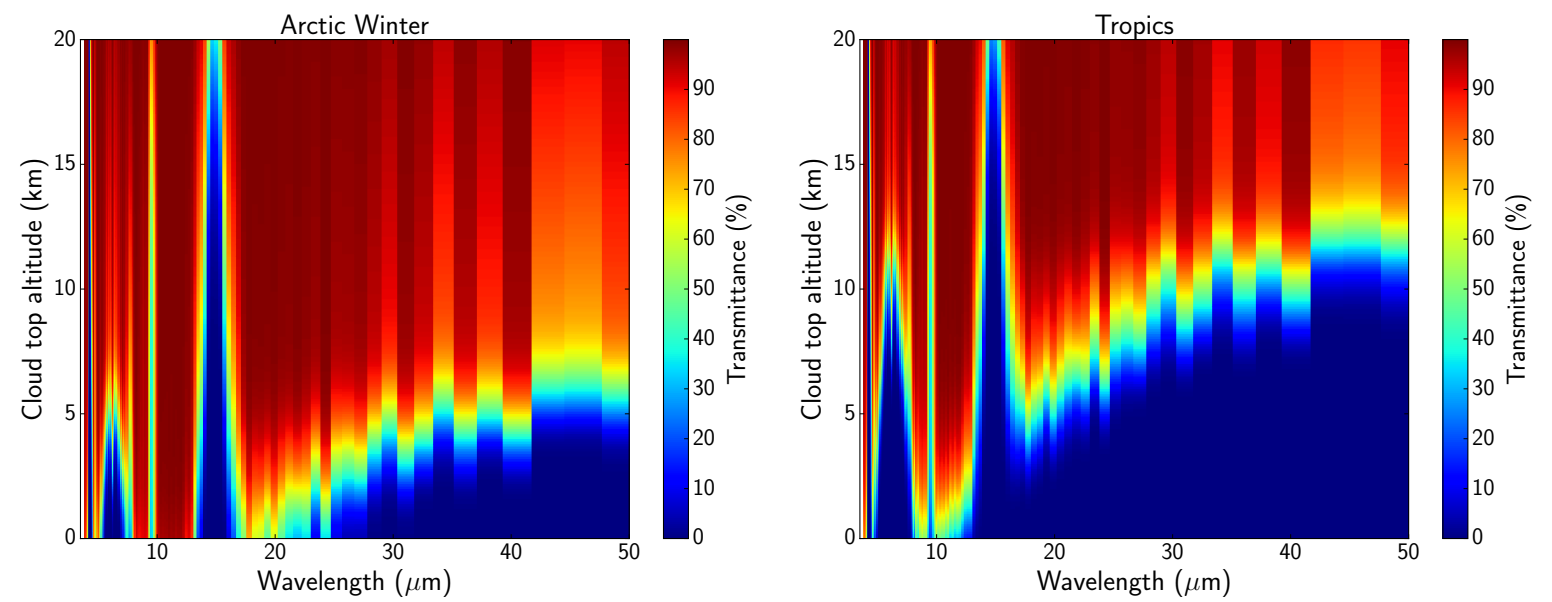

Figure 4. Simulated spectral atmospheric transmittance between the top of a cloud and the TOA as a $15 \mathrm{~cm}^{-1}$ spectral resolution. 

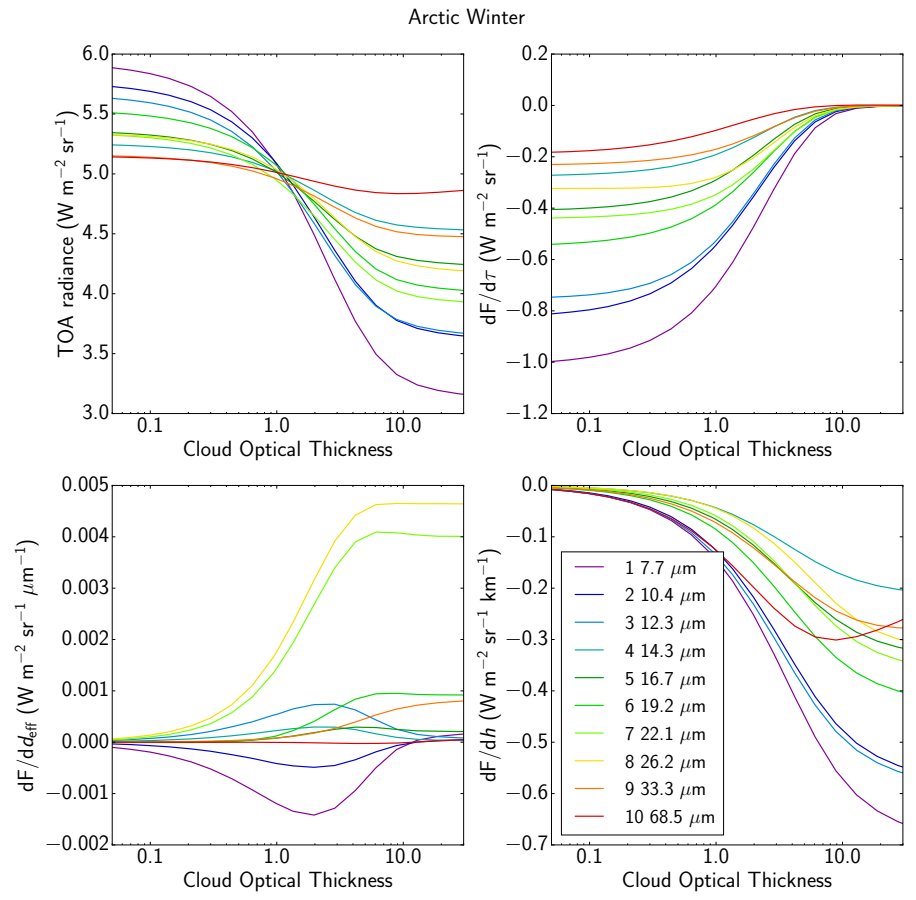

Tropics
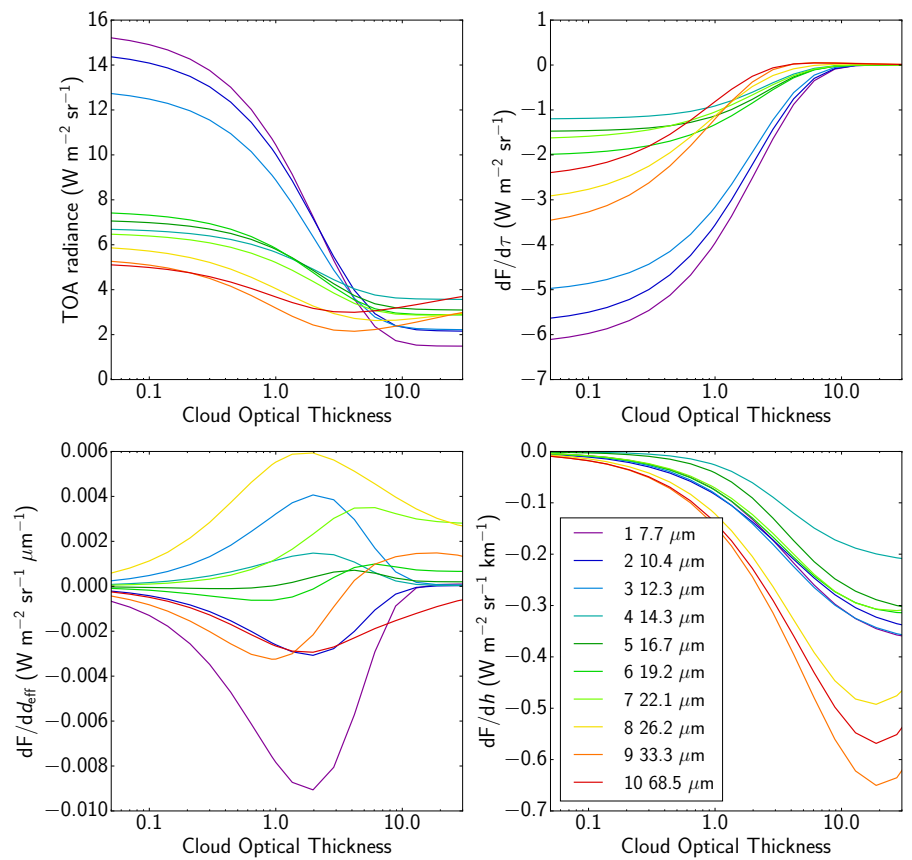

Figure 5. Jacobians of TOA radiance with respect to cloud state parameters for the Arctic winter and tropical atmospheres. Both clouds consist of severely roughened column aggregates with effective diameter $60 \mu \mathrm{m}$ following a modified gamma distribution with effective variance 0.1 . The Arctic winter cloud is located between 5 and $6 \mathrm{~km}$, and the tropical cloud is between 14 and $15 \mathrm{~km}$. 

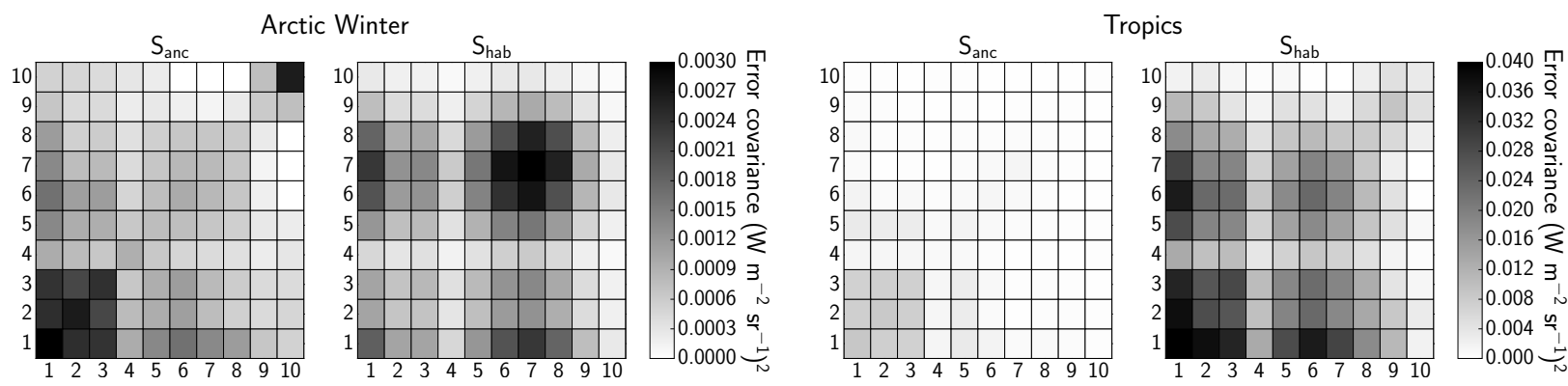
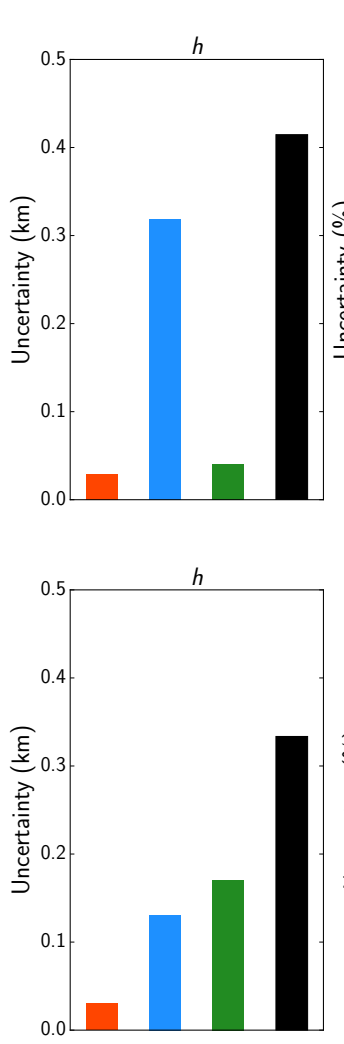
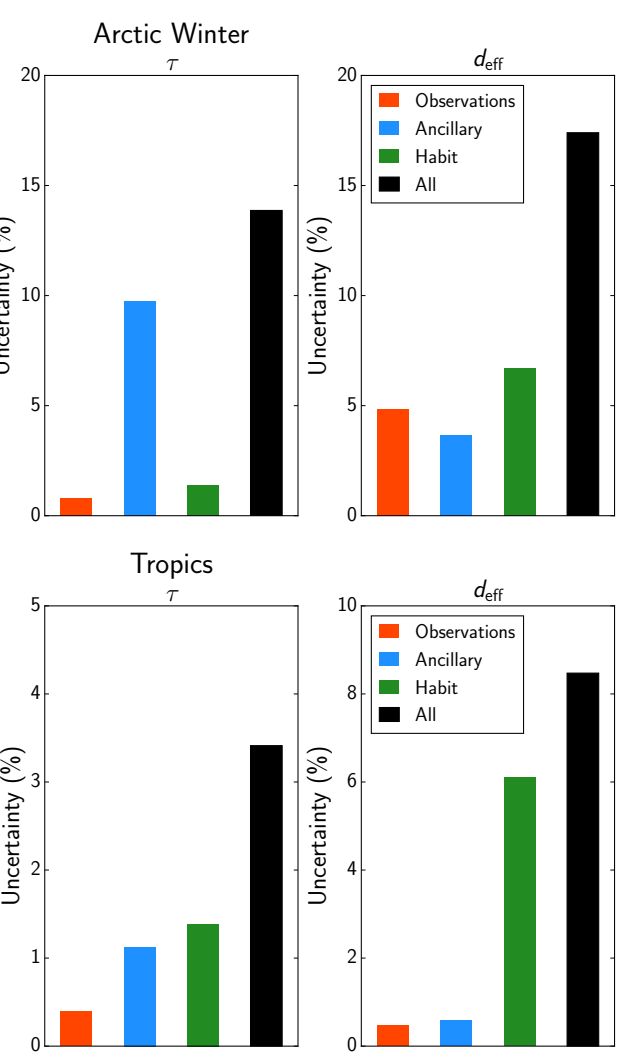

Figure 6. Error covariance matrices of the ancillary parameters $\left(\mathbf{S}_{\mathrm{anc}}\right)$ and crystal habit $\left(\mathbf{S}_{\mathrm{hab}}\right)$ for both reference cases. The numbers correspond to the spectral bands as follows; 1: $7.7 \mu \mathrm{m} ; 2: 10.4 \mu \mathrm{m} ; 3: 12.3 \mu \mathrm{m}$; 4: $14.3 \mu \mathrm{m} ; 5: 16.7 \mu \mathrm{m}$; 6: $19.2 \mu \mathrm{m} ; 7: 22.1 \mu \mathrm{m} ; 8: 26.2 \mu \mathrm{m}$; 9: $33.3 \mu \mathrm{m} ; 10: 68.5 \mu \mathrm{m}$.

Figure 7. Contributions of the error sources to the total posterior uncertainties, when MIR and FIR channels are used. Each source contribution is obtained by computing the difference between the total posterior uncertainties and the uncertainties when this source is removed. 

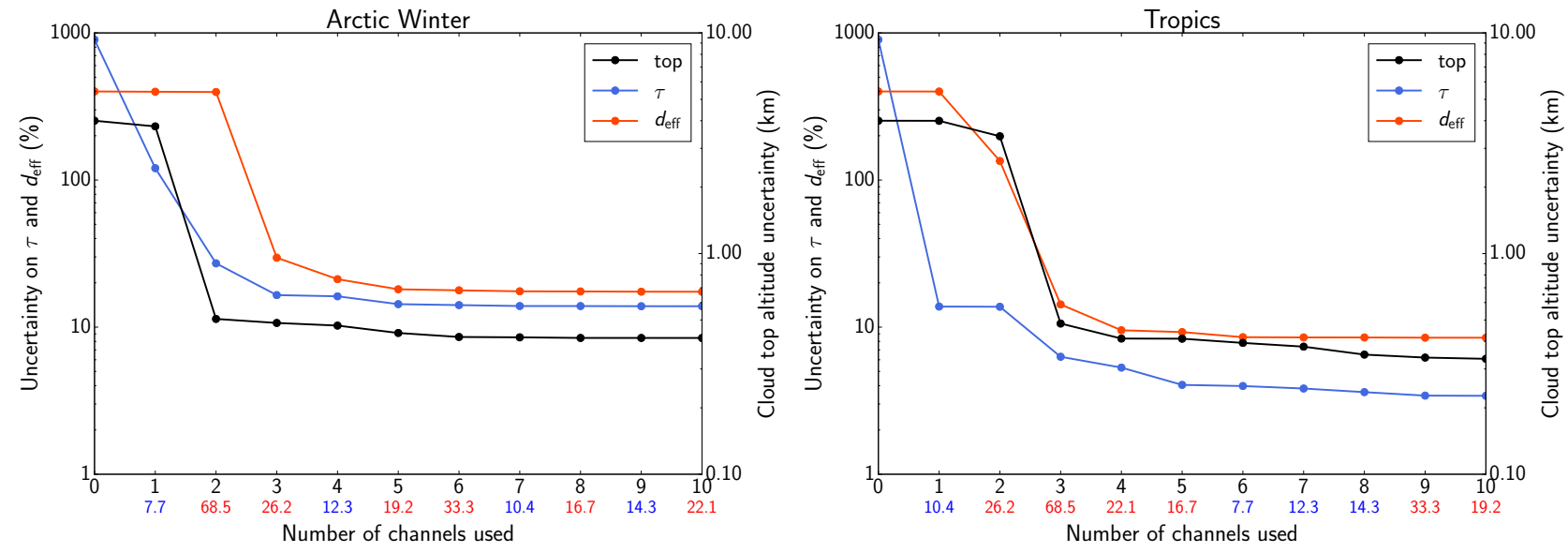

Figure 8. Decrease of state parameters uncertainties as the number of observations increases. The numbers below the graphs indicate the central wavelengths of the selected channels. Blue refers to MIR channels while red indicates FIR channels. 

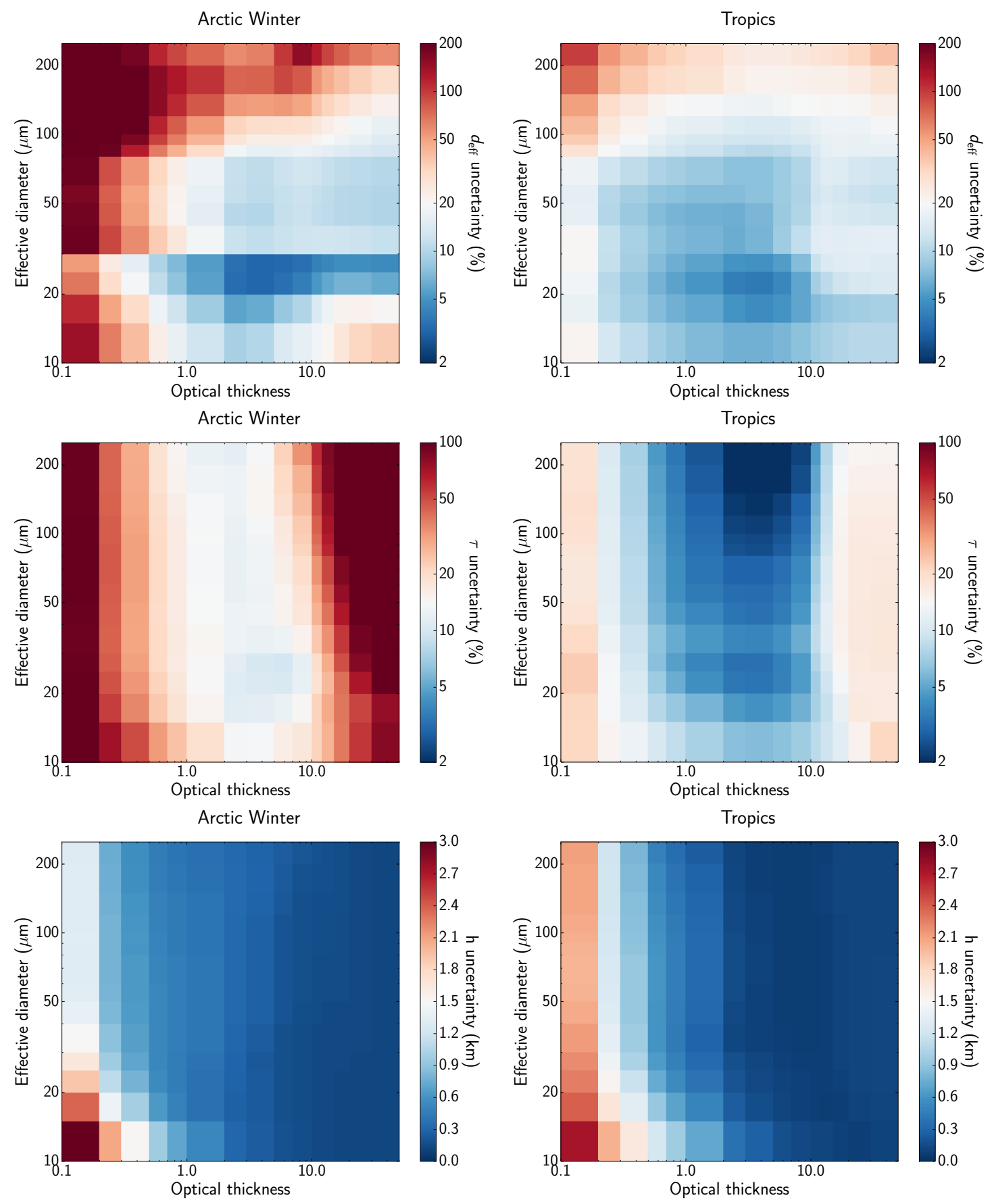

Figure 9. Uncertainties on the $h, \tau$ and $d_{\text {eff }}$ after all channels of the 10-band synthetic radiometer have been used, for a wide range of cloud optical thickness and particle effective diameter. 

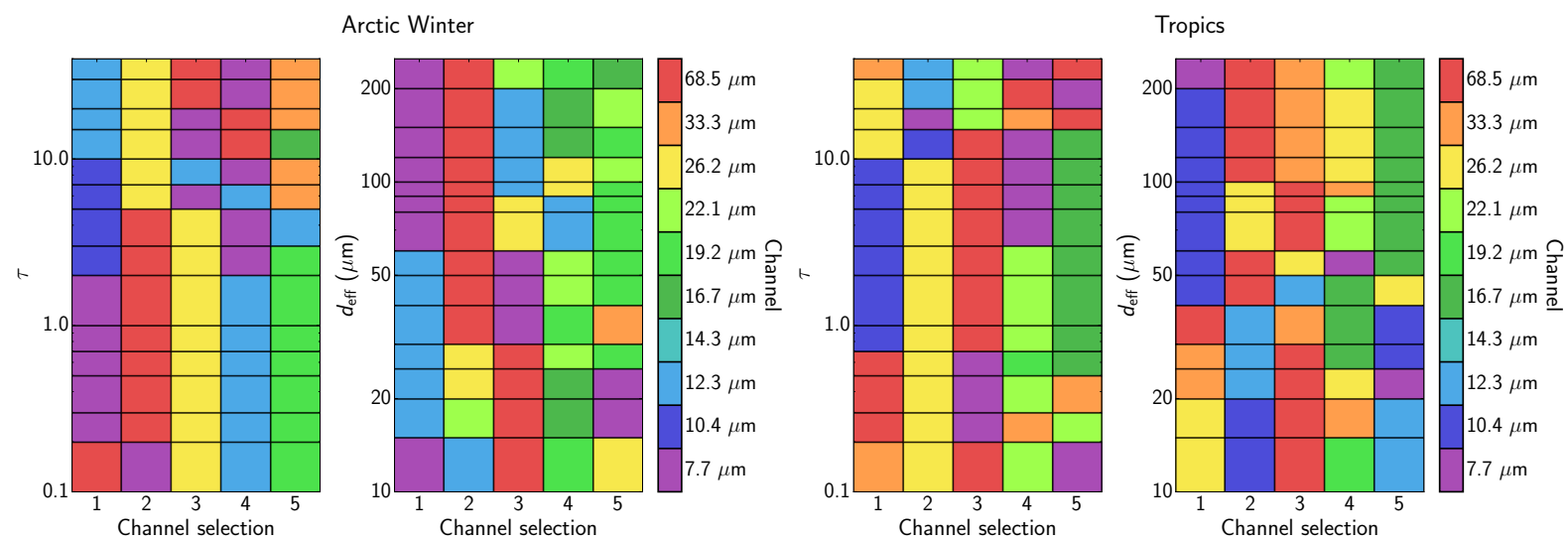
cloud optical thickness (left) or particle effective diameter (right) are varied from the reference cases.
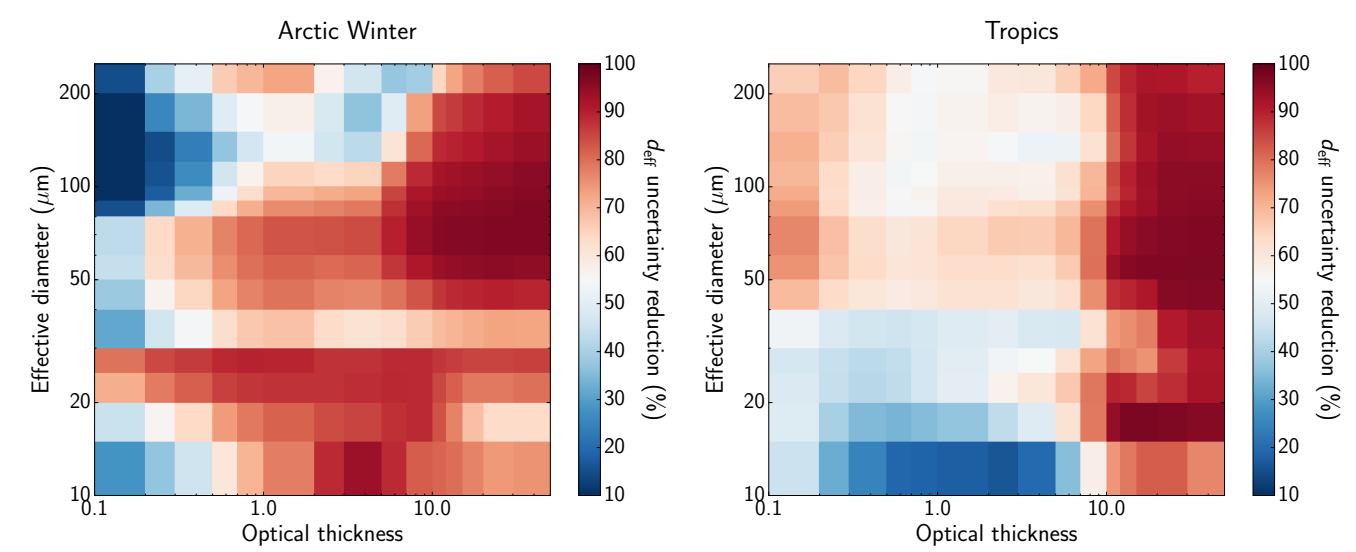

Figure 11. Relative decrease in $d_{\text {eff }}$ uncertainty resulting from the addition of 6 FIR channels to 4 MIR channels, for Arctic winter and tropical conditions. 

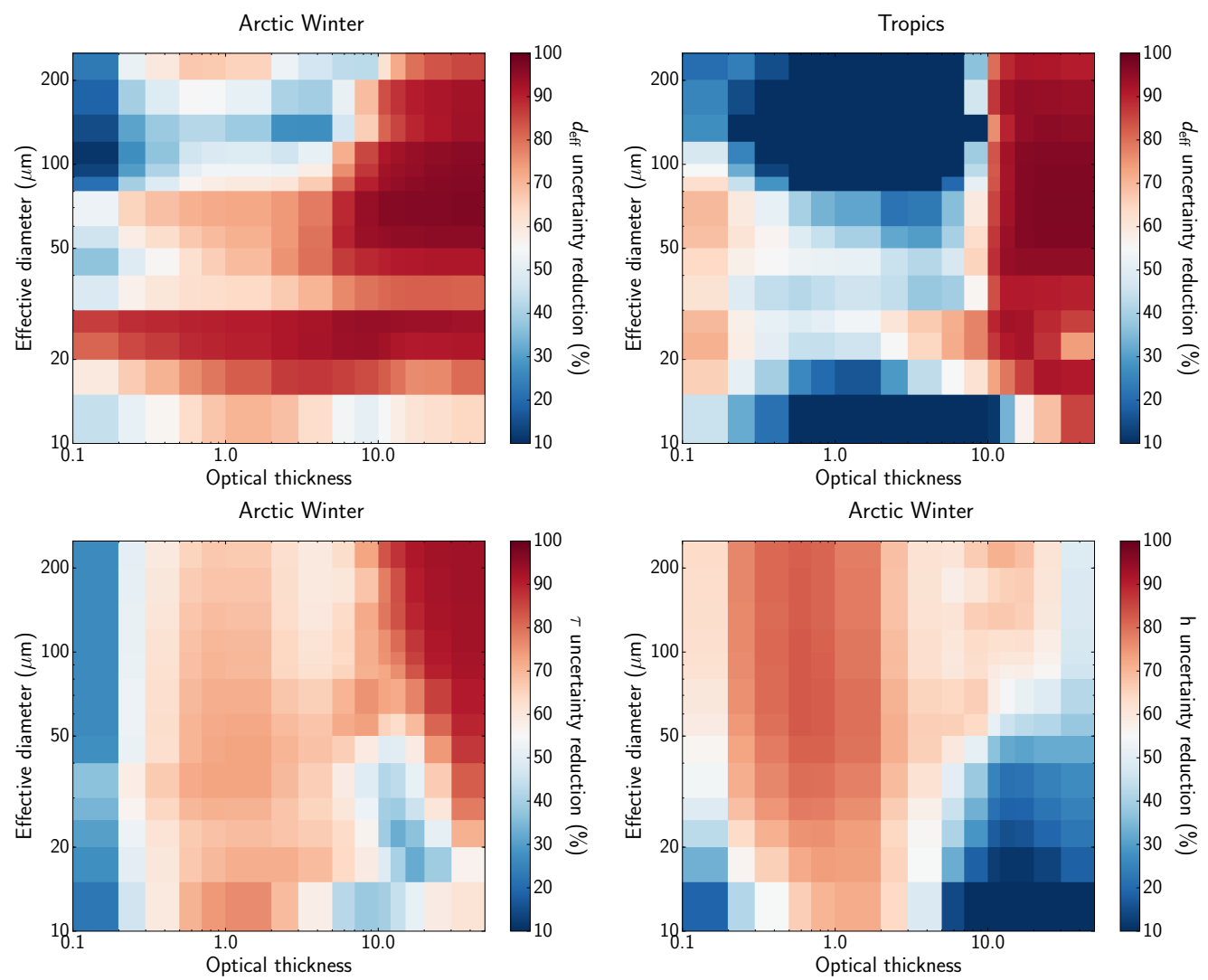

Figure 12. (top) Relative decrease in $d_{\text {eff }}$ uncertainty resulting from the addition of the 9 TICFIRE channels to 10 MODIS channels, for Arctic winter and tropical conditions. (bottom) Relative decrease in $\tau$ and $h$ uncertainties resulting from the addition of the 9 TICFIRE channels to 10 MODIS channels for the Arctic winter atmosphere. 

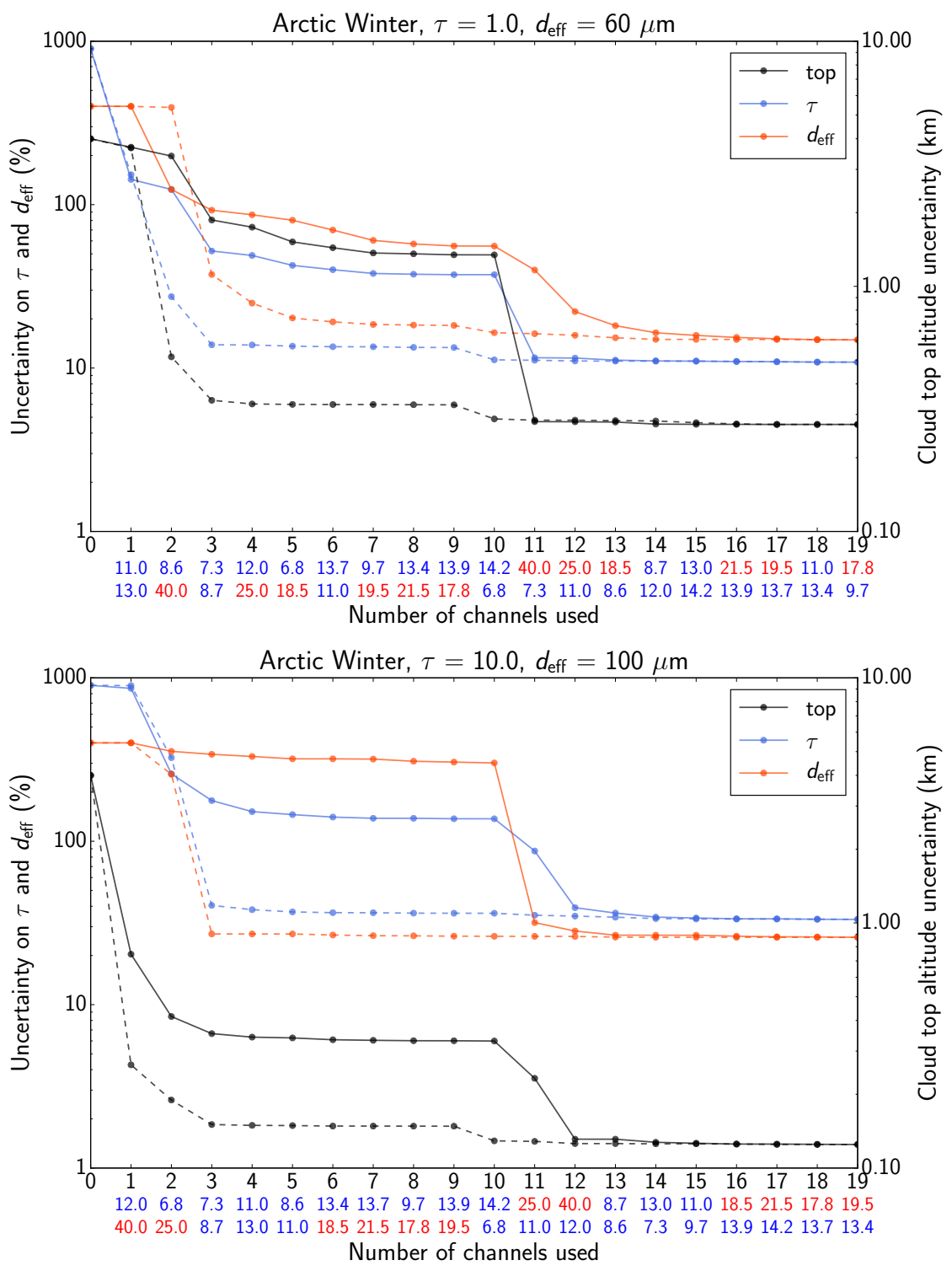

Figure 13. Reduction of cloud properties uncertainties as the number of combined MODIS and TICFIRE observations increases. The plain lines correspond to the case when the 10 MODIS channels are taken first, and then the 9 TICFIRE channels are added. The dashed lines correspond to the case when TICFIRE channels are used first. The numbers below the graphs indicate the central wavelengths of MODIS and TICFIRE channels. The first (respectively second) row corresponds to the plain (respectively dashed) lines. Blue refers to MIR channels while red indicates FIR channels. Note that the TICFIRE instrument has 3 MIR channels and 6 FIR channels. 

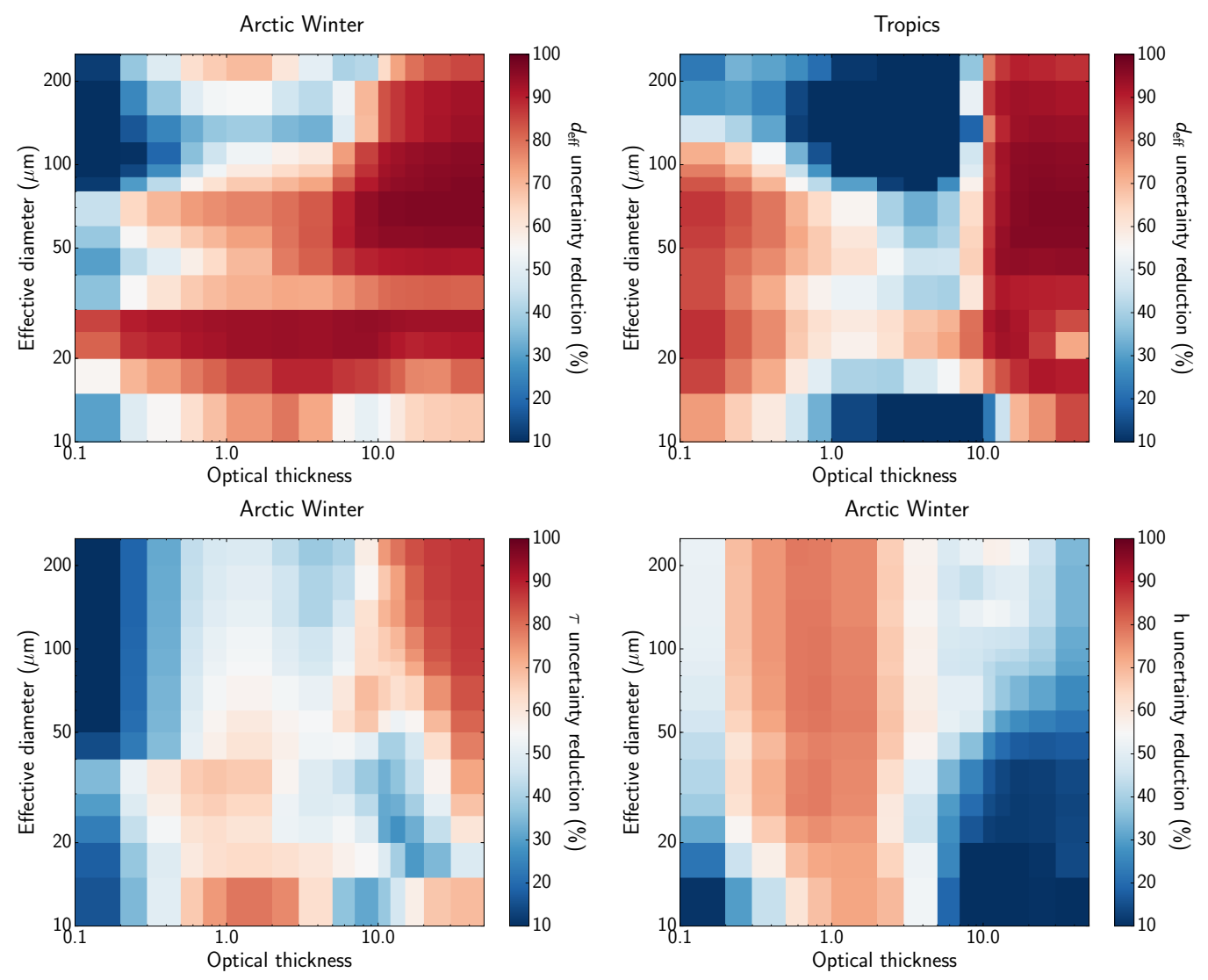

Figure 14. (top) Same as Fig. 12, except that the uncertainties on the ancillary parameters are larger (see Table 3). 

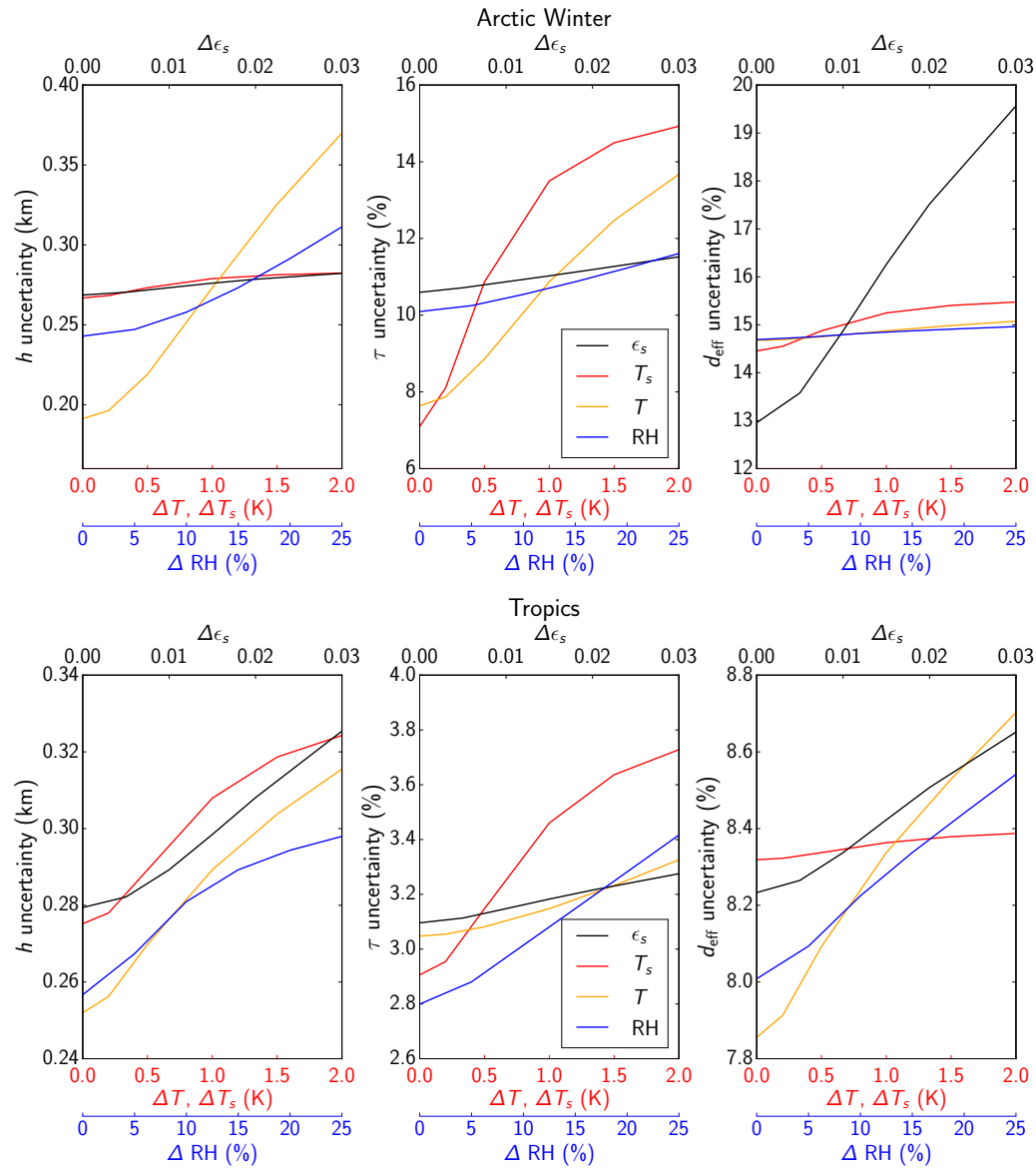

Figure 15. Variations of the posterior state parameters uncertainties as a function of the ancillary parameters errors for the two reference cases. When the uncertainty of one parameter is varied, all others are maintained at their nominal values (see Table 3). 

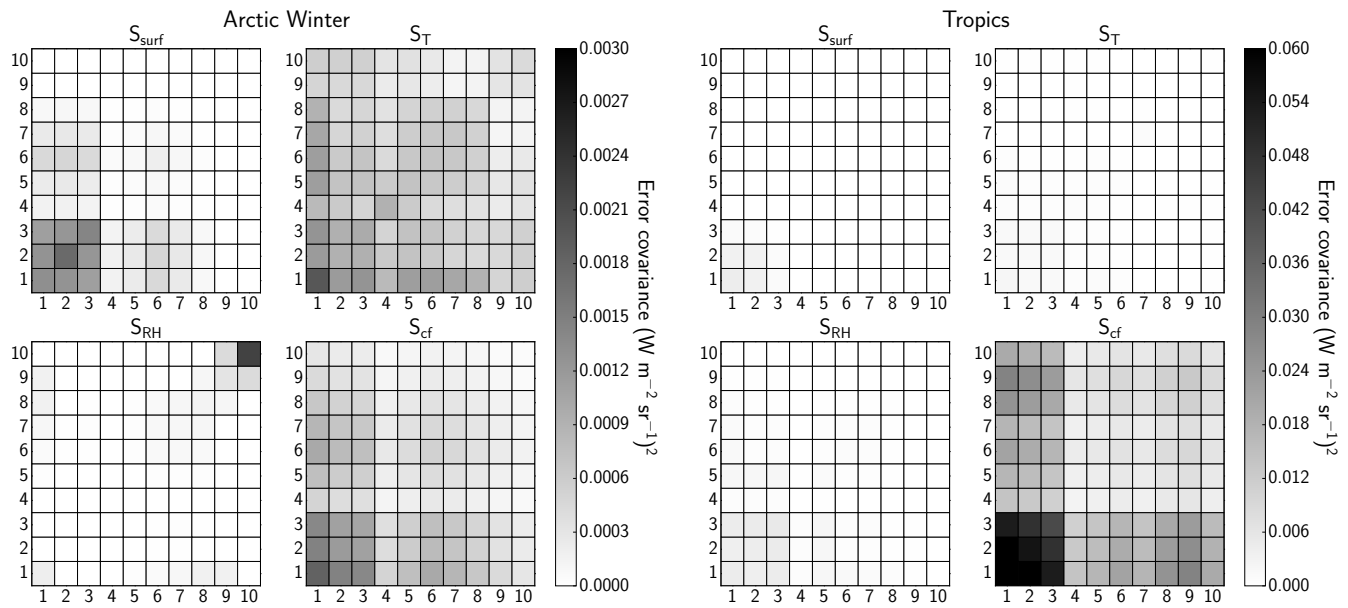

Figure 16. Error covariance matrices associated to the ancillary parameters $\left(\mathbf{S}_{\mathrm{surf}}, \mathbf{S}_{\mathrm{T}}\right.$ and $\left.\mathbf{S}_{\mathrm{RH}}\right)$ and to the uncertainty on cloud fraction $\left(\mathbf{S}_{\mathrm{cf}}\right)$ for both reference cases. $\mathbf{S}_{\mathrm{surf}}$ is the sum of the surface emissivity and surface temperature errors. The numbers correspond to the spectral bands as follows; $1: 7.7 \mu \mathrm{m} ; 2: 10.4 \mu \mathrm{m}$; 3: $12.3 \mu \mathrm{m} ; 4: 14.3 \mu \mathrm{m} ; 5: 16.7 \mu \mathrm{m} ; 6: 19.2 \mu \mathrm{m} ; 7: 22.1 \mu \mathrm{m} ; 8: 26.2 \mu \mathrm{m} ; 9: 33.3 \mu \mathrm{m} ; 10: 68.5 \mu \mathrm{m}$.

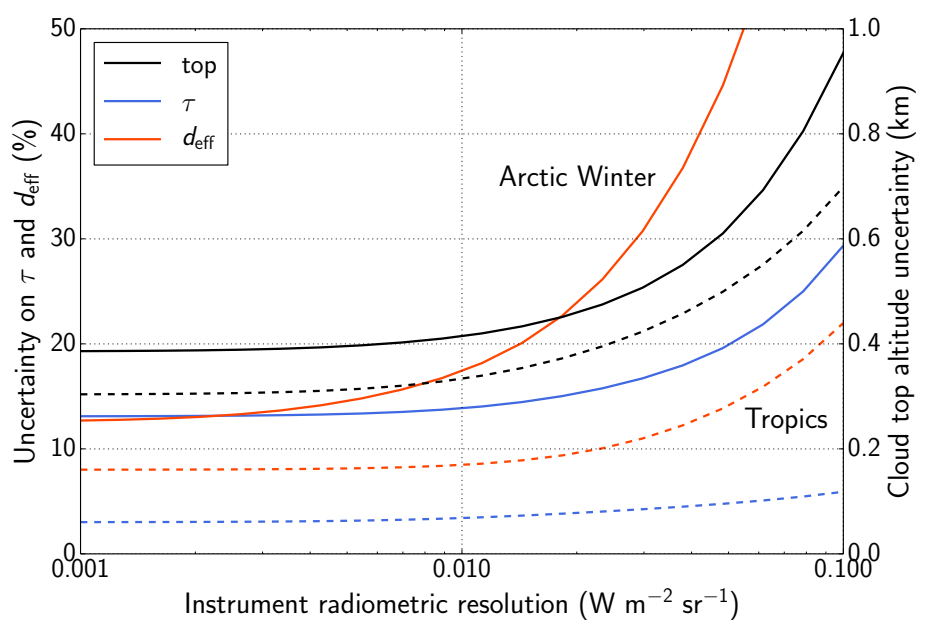

Figure 17. Variations of cloud retrieval uncertainties as a function of instrument radiometric resolution, for the two reference cases. The plain lines correspond to the Arctic winter case, and the dashed lines to the tropical case. 
(a)

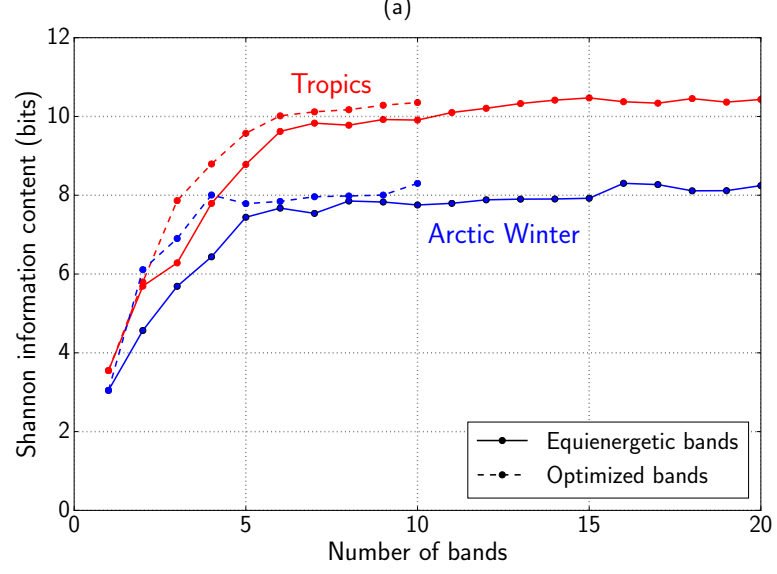

(b)

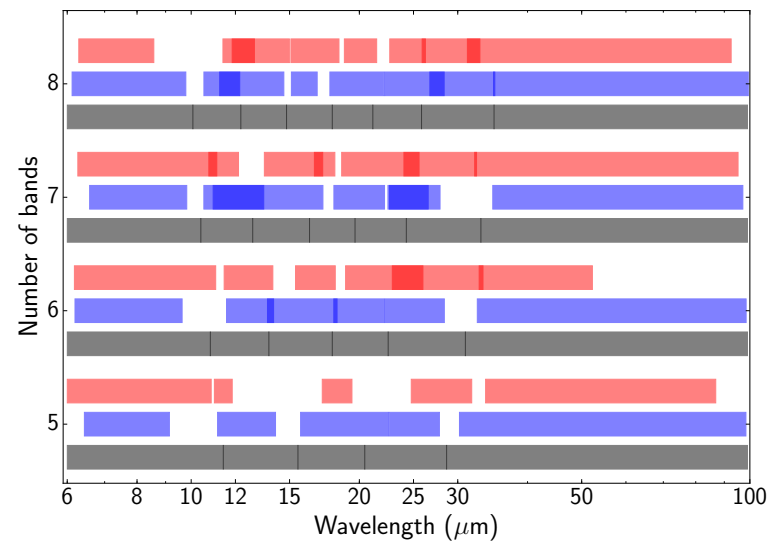

Figure 18. (a) Shannon information content of the observations as a function of the total number of bands, for the two reference cases. The plain lines corresponds to equienergetic bands, while the dotted lines correspond to optimized bands. (b) Spectral characteristics of the equienergetic and optimized bands for the Arctic (blue) and tropical (red) reference cases, for the 5, 6,7, and 8-band configurations. 